\title{
Crecimiento económico en Colombia: una aproximación empírica fundamentada en la perspectiva capital humano (1960-2009)
}

Economic growth in Colombia: an empirical approximation founded in human capital (1960-2009)

María Esperanza Cuenca Coral * Ignacio Penagos Montoya**

Fecha de recepción: 1 de julio de 2014

Concepto de evaluación: 4 de agosto de 2014

Fecha de aprobación: 15 de septiembre de 2014

* Magíster en Economía Pontificia Universidad Javeriana. Economista Universidad Nacional. Bogotá, Colombia. Docente del programa de economía de la Pontificia Universidad Javeriana. Bogotá, Colombia. Correo electrónico: mcuenca@ javeriana.edu.co.

* Economista y Magíster en Economía Pontificia Universidad Javeriana. Docente de planta de la Pontificia Universidad Javeriana. Bogotá, Colombia. Correo electrónico: ipenagos@javeriana.edu.co 
Crecimiento económico en Colombia: una aproximación empírica fundamentada en la perspectiva ...

María Esperanza Cuenca Coral - Ignacio Penagos Montoya

\section{Resumen}

El objetivo de este artículo es especificar un modelo empírico basado en los argumentos teóricos, que cuantifique la influencia del capital humano, medido a través de un conjunto de variables que reflejan el comportamiento de la salud y la educación, sobre el resultado del crecimiento económico observado para Colombia, entre los años 1960 y 2009. Realiza un recorrido por la literatura referente al crecimiento económico y el capital humano, representado por la salud y la educación, de tal manera que, con las fuentes de información disponibles en Colombia, realiza una selección de datos que permite la obtención de variables que reflejen y cuantifiquen los canales de trasmisión sugeridos por la teoría existente. Para terminar, especifica un modelo empírico que estima la relación teórica entre el capital humano y el crecimiento económico.

Palabras clave: crecimiento económico, salud, educación, capital humano.

JEL: E24, E25, I19, I29, O4, O49.

\section{Abstract}

The aim of this paper is to specify an empirical model based on theoretical arguments, quantifying the influence of human capital, measured through a set of variables that reflect the health and education behavior on the outcome of economic growth observed for Colombia, between 1960 and 2009. The authors review the literature on economic growth and human capital, represented by health and education, so that, with the sources of information available in Colombia, they do a selection of data that allows the collection of variables that reflect and quantify the transmission channels suggested by existing theory. Finally, they specify an empirical model that estimates the theoretical relationship between human capital and economic growth.

Keywords: economic growth, health, education, human capital. 


\section{INTRODUCCIÓN}

El concepto y la importancia del capital humano son abordados desde múltiples disciplinas y con criterios diversos; de hecho, no es nuevo en el ámbito de los estudios que tratan de establecer su influencia en el crecimiento económico. Ya hacia mediados del siglo XX, Theodore Schultz (1972) y Gary Becker (1983) planteaban la existencia de un concepto de capital humano, que sería retomado por autores como Mincer (1984), en estudios posteriores. El capital humano no es un descubrimiento reciente en la literatura; su estudio nace a finales de la Segunda Guerra Mundial, cuando es necesario reconstruir las economías y generar condiciones que garanticen prosperidad. En efecto, es hacia 1950 que se genera toda una corriente de pensamiento que pretende aplicar la investigación al crecimiento económico y a la distribución del ingreso. Las tendencias de estudio actuales lo han retomado en diversas formas, relacionándolo además con el concepto de crecimiento económico, en estudios tan importantes como el de Romer (1994).

El objetivo de este artículo es especificar un modelo empírico basado en los argumentos teóricos existentes en la literatura que cuantifique la influencia del capital humano, medido a través de un conjunto de variables que reflejen el comportamiento de la salud y la educación, sobre el resultado del crecimiento económico observado para Colombia, entre los años 1960 y 2009, sujeto a la disponibilidad de datos. Esta aproximación se lleva a cabo siguiendo los lineamientos de Lucas (1988), dentro de los cuales no se busca realizar un análisis de los factores determinantes últimos del crecimiento, pues se busca 
hacer una aproximación a la contabilidad de los aportes que cada uno de los factores relacionados con el capital humano tiene sobre el crecimiento.

Para comenzar, se hace un recorrido por la literatura referente al crecimiento económico y al capital humano, representado por la salud y la educación, que permita deducir los canales y mecanismos de transmisión apropiados que los relacionan. Además, a partir de las fuentes de información en Colombia, se lleva a cabo una selección de datos que permita la obtención de variables que reflejen los canales de trasmisión sugeridos por la teoría existente, usando bases de datos e informes oficiales y así, por último, especificar un modelo empírico para Colombia, con el fin de estimar la relación entre el capital humano y el crecimiento económico, controlando su efecto a través de variables tradicionales como el crecimiento en el stock de capital físico, entre otras.

La hipótesis central de este trabajo sostiene que el capital humano, que incorpora la educación y la salud, tiene una importancia significativa en el resultado del crecimiento económico de Colombia para la época de estudio fijada. Al mismo tiempo, que entre crecimiento económico, educación y salud existen relaciones en un doble sentido, después de controlar sus efectos a través de otras variables, sobre las que, tradicionalmente, se ha considerado que pueden afectar al primero. El trabajo busca aportar en el sentido de resaltar la importancia de la incorporación de la salud en los estudios de capital humano para Colombia, como complemento de la educación, que se encuentra presente en la mayoría de trabajos.

\section{ANTECEDENTES Y PLANTEA- MIENTO DE LA INVESTIGACIÓN}

Los primeros desarrollos dentro del estudio del crecimiento económico de manera formal, dentro de la tradición neoclásica, están relacionados con el modelo de Harrod-Domar (1939), quienes, a partir de una función de producción de proporciones fijas, plantean un modelo en el cual las variaciones de los insumos proveen el crecimiento del producto. Cabe resaltar tres elementos claves dentro de este modelo: el crecimiento garantizado, que es el que los productores conocen sin necesidad de realizar inversiones adicionales; el crecimiento natural, dado por la expansión de la mano de obra por el crecimiento de la población; y por último, el crecimiento actual, que corresponde a la medición del crecimiento en cada momento del tiempo.

A propósito, Solow (1988) muestra la conclusión más relevante acerca de este modelo y tal vez su mayor debilidad

(...) Harrod y Domar obtenían su resultado bajo el supuesto de que los tres ingredientes fundamentales -la tasa de ahorro, la tasa de crecimiento de la fuerza de trabajo y la razón capital/ producto- eran constantes dadas, hechos de la naturaleza. La tasa de ahorro era un hecho de las 
preferencias; la tasa de crecimiento de la oferta de mano de obra era un hecho demográfico- sociológico; la razón de capital/producto era un hecho tecnológico.

Una de las implicaciones más importantes de este modelo es que si la economía cambiaba sobre la recta de equilibrio de la misma, es posible que ya no tienda a él. Es por eso que algunos autores lo denominan el modelo del filo de la navaja. A partir de este modelo surgen dos grandes modelos: el de Kaldor (1955), el de Solow (1956), cuyas diferencias están, básicamente, en los supuestos y resultados obtenidos, pues en el caso del de Solow algunas de las caracterizaciones de la función de producción corresponden a los postulados de la escuela neoclásica.

Los modelos iniciales de crecimiento económico, se caracterizaron por la existencia de dos tipos de planteamientos, en cuanto a la forma como se trata el ahorro: los de tasas de ahorro constante y los de senda de ahorro óptima. La forma como se aborda el ahorro -al ser considerado este como una variable relevante en el análisis- determina el tipo de modelo resultante. El objetivo es explicar de qué dependen las variaciones del ingreso a través del tiempo. Casi de manera contemporánea, la necesidad de entender el capital humano surge alrededor de 1950 , a partir de estudios empíricos acerca del crecimiento económico y la distribución del ingreso. Nace bajo dos esquemas alternativos de estudio: por una parte, el enfoque macroeconómico, en el cual se relaciona con los agregados y el crecimiento económico; el segundo enfoque posible es el microeconómico, donde los individuos realizan cálculos para medir la incidencia de educación en sus salarios y en la distribución del ingreso.

Dentro de la evolución de las ideas acerca del crecimiento, se han abierto varios campos de estudio, siguiendo con los principios básicos de Arrow (1962), Romer (1994) y con base en la evidencia las externalidades que, sobre otras empresas y en general sobre la economía, tienen los descubrimientos de una firma. Trabajos posteriores de Romer (1986) y Lucas (1988) incorporan la idea del crecimiento endógeno, el cual encierra un cuerpo diverso de trabajos empíricos y teóricos que emergió en los años ochenta diferenciándose del crecimiento neoclásico, pues hace énfasis en que el crecimiento económico es un resultado endógeno de un sistema económico, no el resultado de fuerzas que lo impulsan desde afuera.

Ahora bien, siguiendo a Mincer (1993), existen dos maneras posibles de comprender el capital humano; la primera, desde el punto de vista macroeconómico, entendiendo su relación con el crecimiento; o desde la perspectiva microeconómica, cuando se toma en consideración cómo mayores inversiones en educación modifican la estructura salarial y la distribución individual del ingreso.

Uno de los modelos más importantes en cuanto al enfoque macroeconómico es el de Lucas (1988). La implicación del 
efecto externo en el modelo es que, bajo un equilibrio puramente competitivo, su presencia conduce a una subinversión en capital humano, puesto que la educación genera una externalidad que no es tenida en cuenta por los agentes privados. Así pues, la tasa de crecimiento de equilibrio es menor que la tasa de crecimiento óptima, debido a que la tasa de crecimiento de equilibrio depende de la inversión en capital humano, por lo tanto, se concluye que se requieren políticas gubernamentales (subsidios) para incrementar la tasa de crecimiento de equilibrio hasta la tasa de crecimiento óptima.

En general, las externalidades que más llaman la atención en los estudios son las generadas por la educación, pero en trabajos recientes ha cobrado importancia el efecto de la salud sobre el crecimiento económico. Weil (2005) utiliza un modelo microeconométrico para estimar el efecto de la salud en el PIB per cápita, controlado por una variable de educación en una muestra de países, en la que se encontró que alrededor del $22.6 \%$ de la varianza del logaritmo del PIB entre países está explicada por su nivel de salud, participación similar a la varianza explicada por las diferencias en el nivel de educación.

Por ahora se dirá que el capital humano no es un descubrimiento reciente en la literatura, a pesar de que su desarrollo sí lo es. Siguiendo a Jiménez y Simón (2004),

Hace más de 200 años que Adam Smith (1776) reconoció la importancia de las habilidades personales en la determinación de la riqueza de los individuos y de las naciones. Sin embargo, el concepto formal de capital humano no fue desarrollado hasta las décadas de 1960 y 1970 con los trabajos de Schultz (1960,1961), Becker (1962,1964) y Blaug (1976). En ellos, el capital humano se relaciona con la productividad y es definido como la suma de las inversiones en educación, formación en el trabajo, emigración o salud que tiene como consecuencia un aumento en la productividad de los trabajadores.

Según Gaviria (2007), la "nueva" teoría del crecimiento pretende romper con la versión tradicional de los modelos neoclásicos (Solow-Swan), según la cual, las tasas de crecimiento del producto y de la población se igualan en el estado estacionario. De la misma manera, busca endogenizar el cambio técnico, atando su evolución al capital físico. Más adelante afirma que en el artículo que dio origen a la literatura del crecimiento endógeno, Paul Romer (1986) eliminó la tendencia de los rendimientos decrecientes del capital, al suponer que el conocimiento era obtenido como un subproducto de la inversión en capital físico. Este fenómeno es conocido como aprendizaje por la práctica (learning-by-doing) y fue planteado inicialmente por autores como Arrow (1962) quien afirmó que el progreso técnico presentaba un comportamiento endógeno.

Así mismo, aparece evidencia internacional de la importancia del capital humano 
en el crecimiento: un alto nivel educativo permite una mejor adaptación de las tecnologías, así como un mayor acceso de la mayoría de la población a mejores niveles de vida. Con respecto a dicho capital, también puede decirse, como Posada y Rubiano (2007), que el crecimiento económico, independiente de su causa, genera un aumento en los retornos al capital humano, por lo cual aumenta su acumulación, entre otras cosas, debido a que las nuevas tecnologías requieren habilidades y conocimientos nuevos y avanzados de quienes están inmersos en el sistema productivo.

Esta última inclusión aparece en varios trabajos de Weil (2005) o en el trabajo más reciente de Aghion, Howitt y Murtin (2010), donde los autores postulan que la intuición económica, apoyada en evidencia empírica particular, sugiere que la salud incide en el crecimiento a través de por lo menos dos canales. En primer lugar, los individuos con mayor esperanza de vida es probable que ahorren más, lo cual favorece la acumulación de capital y por lo tanto el crecimiento del PIB. En segundo lugar, los individuos con mayor esperanza de vida es probable que inviertan más (o que sus padres invierten más) en la educación, lo cual genera crecimiento. Ahora bien, en un entorno marcado por la mortalidad infantil baja, los padres procuran un bajo nivel de fertilidad, lo que limita el crecimiento de la población total e impulsa el crecimiento del PIB per cápita. Por último, y más directamente, los individuos sanos suelen ser más productivos y más capaces en la creación y adaptación a las nuevas tecnologías y, en general, más capaces de hacer frente a los rápidos cambios característicos de un entorno de alto crecimiento.

La mayor dificultad al querer realizar este análisis de la manera más completa posible es que las relaciones no son unívocas entre crecimiento, educación y salud. La percepción de la educación ha cambiado en la teoría económica; en la teoría neoclásica se consideraba como un insumo de la producción; en los sesenta, se incorporó en los modelos de crecimiento en el residuo de Solow. Los individuos nacen con una dotación de ciertas habilidades y capacidades, pero la educación puede potenciarlas y mejorar el desempeño laboral. Ahora bien, Grooth y Massen (1993) han establecido que la productividad depende de la educación de los individuos y de su estado general de salud, y se sabe que el nivel de educación incide en la salud.

En este estudio se busca, a través del modelo econométrico resultante, involucrar estas interrelaciones y que se pueda evidenciar la contribución del capital humano en el crecimiento económico colombiano. Trabajos similares son, por ejemplo, el de Whalley y Zhao (2010), quienes realizan un estudio acerca de la contribución del capital humano en el crecimiento chino, en el que encontraron que durante el periodo 19782008 el stock de capital humano creció anualmente $7.6 \%$ y contribuyó $33.2 \%$ al crecimiento.

Este trabajo incorpora la salud y la educación como insumos de la función de 
producción, siguiendo a Lucas (1988), a través de los resultados del mejoramiento en una de estas variables. Es decir, la salud, por ejemplo, se incluye a través de las mejoras en salud que se reflejan, entre otros aspectos, en la esperanza de vida, la tasa de natalidad y mortalidad.

\section{Planteamiento del problema y canales de transmisión}

Partiendo del análisis teórico hecho por múltiples autores como Mincer (1993), la salud comienza a tener influencia en el desempeño futuro de un trabajador desde el momento de su gestación. Las mejoras en salud de las madres gestantes, el cuidado de la salud infantil y el nivel de educación de los padres, entre otros factores socio-económicos, junto con la "dotación inicial" de habilidades del recién nacido, influyen en el desarrollo del niño, y en el estatus de salud y las decisiones sobre educación que tomará el adolescente.

Individuos más saludables tienden a tener una mayor expectativa de vida (Mincer, 1993), por lo que los retornos a la educación aumentan y hacen más atractiva la opción de elegir una mayor cantidad de años de la misma. Por otro lado, individuos más saludables en la adolescencia son adultos más sanos y más productivos en sus trabajos. De esta manera, salud y educación interrelacionadas propician individuos capaces de una mayor productividad, por lo que la riqueza de un país está determinada no solo por su acumulación de capital físico, sino también por el acceso a educación y el nivel de salud de su población.
Algunos autores como Bils y Klenow (2000) señalan que individuos más educados son individuos más productivos y por lo tanto a través de la productividad se establece el vínculo entre educación y crecimiento económico. Por otra parte, estudios de salud y crecimiento señalan que la salud medida a través de la nutrición tiene un efecto positivo sobre la productividad y por ende en el crecimiento económico. También existen otras investigaciones como la de Cleuter y Lleras-Muney (2006), que intentan comprobar la relación entre la educación y la salud; por ejemplo, individuos más educados son más conscientes de llevar a cabo comportamientos más saludables por lo que, para estos, las tasas de mortalidad suelen ser más bajas, pero también de manera inversa, una mayor expectativa de vida hace que sea más rentable acceder a un mayor nivel de educación, de tal forma que el retorno de la educación aumenta y los individuos más saludables (que esperan vivir más tiempo) están dispuestos a acumular un mayor nivel de años de educación. Lo que queda claro es que tanto la salud como la educación tienen efectos en la productividad y que la salud tiene efectos en la educación y esta última en la primera, como concluyen Groot y Maassen (2007).

Ahora bien, la evidencia empírica ha demostrado que no todos los países tienen la misma dinámica en torno al crecimiento. De hecho, según Agénor y Montiel (2000), los enfoques neoclásicos tradicionales, que atribuyen el crecimiento al progreso tecnológico 
exógeno, no pueden explicar las grandes disparidades del ritmo del crecimiento económico entre los países. En los últimos años se han hecho esfuerzos considerables para entender las fuentes de crecimiento económico y explicar los patrones divergentes observados entre los países. Dentro de esta tendencia teórica, y siguiendo a Agénor y Montiel (2000), una fuente particular de las externalidades que se ha destacado en la literatura del crecimiento reciente es la acumulación de capital humano y su efecto sobre la productividad de la economía. Lucas (1988) ofrece uno de los esfuerzos mejor conocidos para la incorporación de los efectos de filtración de la acumulación de capital humano, en un modelo basado en la idea de que los trabajadores individuales son más productivos, independientemente de su nivel de habilidad, si otros trabajadores tienen más capital humano.

La Figura 1 ilustra la red de interacciones que muestran los diferentes estudios citados. Se debe tener en cuenta que no existe una única forma de transmisión entre una variable y otra. Por ejemplo, los cuidados de la madre gestante afectan la salud del neonato, pero también la van a afectar la nutrición durante su infancia y adolescencia, el nivel y calidad educativa que reciba y las decisiones que en su vida adulta tome sobre la prevención de riesgos en salud, por lo que estos canales de transmisión no son únicos ni contemporáneos, pero sí se pueden evidenciar a través de una serie de variables que muestren el resultado de esta cadena de acciones.

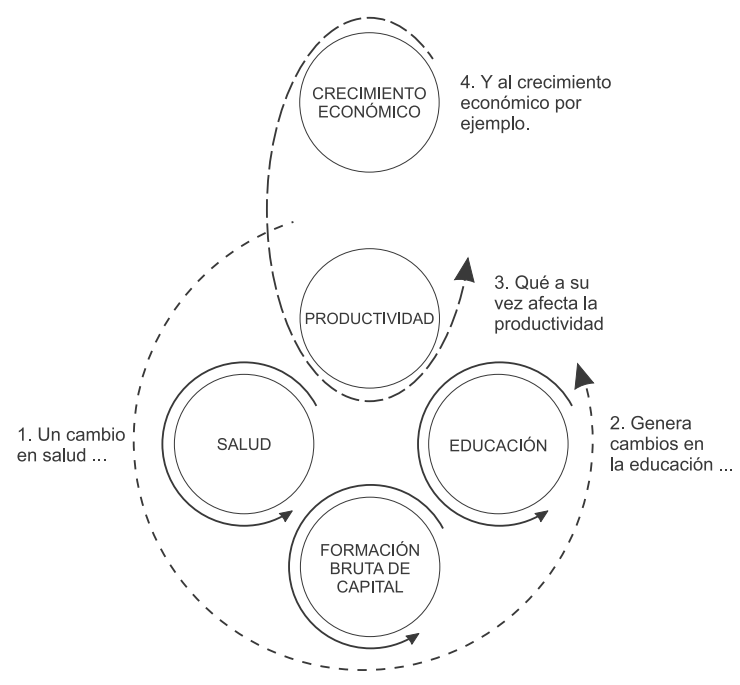

Figura 1. Esquema de relación entre las variables.

Fuente: elaboración de los autores. 
Nótese cómo se conciben las interacciones entre las variables en cuestión: un engranaje donde un cambio en salud puede, además de mejorar la salud misma de los individuos, impulsar a la educación, y a través de la productividad al crecimiento económico y de allí a la acumulación de capital, etc. Y por allí mismo, el incremento en la actividad económica puede devolverle el impulso a la salud en una especie de efecto multiplicador; es decir, que así como el capital humano es un factor de producción, el producto a su vez es un factor en la generación de capital humano, de salud y educación específicamente.

La tasa de mortalidad podría reflejar este resultado, pues, de acuerdo con Weil (2005), de todas las influencias que existan sobre la salud de un individuo a lo largo de su vida y, a su vez, una mejora en la tasa de mortalidad, pueden impulsar a las personas a cambiar sus preferencias de tal modo que se logran modificar los factores que pueden influenciar a la salud y así sucesivamente.

Por lo tanto, un supuesto fundamental que se tendrá es la simultaneidad y las múltiples interacciones entre el producto de la economía, salud y educación y otras variables de control. Un ejemplo de esta concepción es suponer que existe un conjunto de variables que reflejan el estado o nivel de salud. Estas variables pueden ser la incidencia de una cierta enfermedad en la población infantil, la cantidad de calorías consumidas por la población, el peso y la talla promedio de la población, la tasa de mortalidad, etc. A su vez, también existen variables que pueden influenciar el desempeño de las primeras, como el gasto público en salud, el acceso a servicios médicos, el número de camas de hospital, etc.

Por otro lado, las preferencias de los individuos son sensibles a los cambios en salud en interacción con los cambios educativos, como se enunció anteriormente siguiendo a Cleuter y Lleras-Muney (2006) y Groot y Maassen (2007). Si hubiese un incremento en el gasto público en salud que genera una disminución en la incidencia de ciertas enfermedades en la población infantil, este cambio debería incrementar el nivel de salud, lo que a su vez podrá modificar las preferencias de los individuos. Estos cambios en las preferencias pueden presionar a nuevos cambios en las políticas gubernamentales y en los comportamientos propios de los individuos. En suma, existe un número indeterminado de interrelaciones entre variables "input" 1 " y "output" en salud, además de los cambios que se generan en las preferencias de los individuos, los cambios en educación y los cambios en el producto, lo que implica que tanto la educación como la salud asumen dinámicas complejas, tanto autónomas (autocorre-

\footnotetext{
Siguiendo a Weil (2005), son aquellas que en una concepción lineal son insumos para la salud, como el número de camas de hospital o la cantidad de médicos, etc.

2 Según Weil (2005), son las que muestran los resultados en salud, como la tasa de mortalidad, la incidencia de ciertas enfermedades en cierta población, etc.
} 
lacionadas) como interrelacionadas, de forma contemporánea como rezagada en diferentes horizontes temporales.

Dadas las relaciones entre estas variables, se plantea un modelo de vectores autorregresivos, pues una de las ventajas de esta metodología es que permite retratar el hecho de las múltiples relaciones e interacciones entre las diferentes variables. El modelo teórico que se plantea siguiendo la discusión anterior, retratada en la Figura 1, tiene la siguiente forma estructural representada por la ecuación [1]:

$$
\left[\begin{array}{c}
g_{t} \\
K_{t} \\
S A L_{t} \\
E D U_{t}
\end{array}\right]=\left[\begin{array}{c}
\alpha_{g} \\
\alpha_{k} \\
\alpha_{S A L} \\
\alpha_{E D U}
\end{array}\right]+\left[\begin{array}{llll}
\alpha_{1,11} & \alpha_{1,12} & \alpha_{1,13} & \alpha_{1,14} \\
\alpha_{1,21} & \alpha_{1,22} & \alpha_{1,23} & \alpha_{1,24} \\
\alpha_{1,31} & \alpha_{1,32} & \alpha_{1,33} & \alpha_{1,34} \\
\alpha_{1,41} & \alpha_{1,42} & \alpha_{1,43} & \alpha_{1,44}
\end{array}\right]\left[\begin{array}{c}
g_{t-1} \\
K_{t-1} \\
S A L_{t-1} \\
E D U_{t-1}
\end{array}\right]+\ldots+\left[\begin{array}{c}
\varepsilon_{g t} \\
\varepsilon_{k t} \\
\varepsilon_{S A L t} \\
\varepsilon_{E D U t}
\end{array}\right]
$$

Donde $\mathrm{g}_{\mathrm{t}}$ es el crecimiento económico, $\mathrm{K}_{\mathrm{t}}$ es la acumulación de capital EDU es el cambio en el nivel de educación, y $\mathrm{SAL}_{t}$ es el cambio en el nivel de salud para Colombia en un período de tiempo. Se dejan puntos suspensivos que indican la existencia de múltiples rezagos de las cuatro variables, que serán incluidos a fin de explicar los efectos no contemporáneos, y su extensión dependerá de criterios técnicos combinados con criterios teóricos.

\section{CAPITAL HUMANO Y CRECI- MIENTO ECONÓMICO EN CO- LOMBIA (1960-2009)}

A continuación se presentan algunos hechos importantes acerca del crecimiento económico en Colombia, con el fin de mostrar los hechos estilizados que pretenden ser interpretados en el modelo econométrico. En la segunda parte de esta sección aparece una breve descripción de la metodología que se va a emplear y los resultados del modelo.
Para entrar a describir el capital humano en Colombia, se pueden utilizar algunas variables relacionadas con la salud y la educación. Colombia ha sido catalogada como un país en vías de desarrollo. Con relación al índice de desarrollo humano calculado por la Organización de las Naciones Unidas para 2007, el país se encuentra en el puesto 75 con un indicador de 0.791, comparable con Dominica (0.798), Ecuador (0.772), Perú (0.773); pero muy lejos de de Islandia (0.968) o de Sierra Leona (0.336), (los datos extremos de dicho indicador). Su PIB es de alrededor de 234,000 millones de dólares en el 2009, de acuerdo con los datos del Banco Mundial, superior al promedio de los países considerados. Sin embargo, el PIB per cápita está por debajo del promedio mundial. Según Urrutia y Posada (2007), al analizar el último siglo (19002000) de crecimiento económico, puede evidenciarse una relativa estabilidad de la tasa de crecimiento, al compararla con América Latina. 
La dinámica de crecimiento en Colombia ha sido positiva para casi todo el período de la historia económica del país. Entre 1970 y 2005, Colombia, según datos del DANE y los cálculos de los autores, creció en un $4.3 \%$, coherente con la interpretación realizada en algunos análisis previos realizados sobre el tema. Según GRECO (1999), "algunos países avanzaron más rápido que Colombia, y de acuerdo con la literatura de convergencia, en ello influyó no solamente el ingreso per cápita inicial, sino también los niveles iniciales de educación, de infraestructura, de desarrollo institucional, de grado de apertura, etc. (Barro \& Sala-i-Martin (1995)". El estudio realizado por Uribe (1994) encontró además que la inflación tuvo una incidencia negativa sobre el crecimiento en Colombia, sobre todo a partir de 1970 cuando la inflación llegó a ser de dos dígitos.

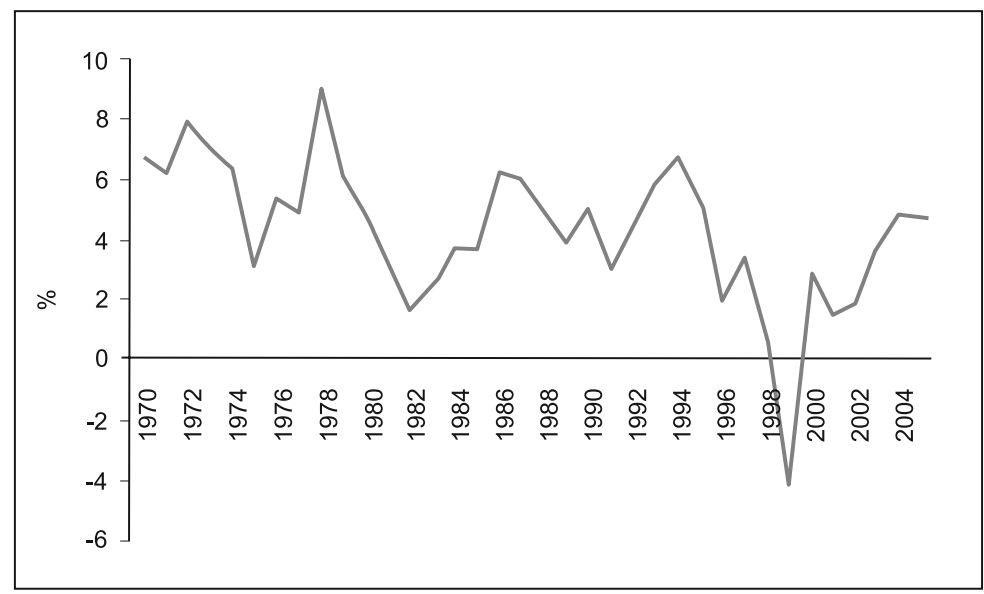

Figura 2. Crecimiento económico en Colombia. Fuente: Dane (2010). Cálculos de los autores.

Esta dinámica del crecimiento puede ser explicada desde diferentes perspectivas. A continuación, y dentro de los objetivos del presente trabajo, se muestra el comportamiento de las variables que se incluirán dentro del concepto de capital humano. Colombia tiene alrededor de 45 millones de habitantes, muchos de ellos aún en las regiones más deshabitadas del país. Las condiciones de salud y educación están limitadas por realida- des diversas; sin embargo existen unos lineamientos generales que serán tenidos en cuenta dentro de este trabajo.

\section{La educación en Colombia}

En la Constitución Política colombiana se indica que la educación es un derecho de la persona, un servicio público que tiene una función social y que corresponde al Estado regular y ejercer la suprema 
inspección y vigilancia respecto del servicio educativo, con el fin de velar por su calidad, por el cumplimiento de sus fines y por la mejor formación moral, intelectual y física de los educandos. También se establece que se debe garantizar el adecuado cubrimiento del servicio y asegurar a los menores las condiciones necesarias para su acceso y permanencia en el sistema educativo. El sistema educativo colombiano está conformado por la educación inicial, la educación preescolar, la educación básica (primaria cinco grados y secundaria cuatro grados), la educación media (dos grados y culmina con el título de bachiller), y la educación superior (Ministerio de Educación, 2009).

Esta estructura, donde además se han descentralizado presupuesto y funciones, ha hecho que la educación en Colombia haya mejorado en cuento a cobertura. Es así como, según datos del Ministerio de Educación Nacional (2009), el número de matriculados en básica secundaria aumentó en más de 300.000 jóvenes entre 2000 y 2005. De acuerdo con el Informe del progreso educativo en Colombia 2006, no obstante, 4 de cada 10 jóvenes en edad de cursar este nivel no estudian; y existen marcadas diferencias entre departamentos: en Vichada, la cobertura es del $21 \%$, mientras que en Bogotá alcanza el $73 \%$. Además, cerca de la tercera parte de los estudiantes tiene un desfase que podría superar en tres o más años la edad esperada para este nivel.

De acuerdo con el Informe nacional sobre educación en Colombia (2001) del Minis- terio de Educación, la educación formal se imparte en establecimientos educativos aprobados, según una secuencia regular de niveles lectivos, con sujeción a pautas curriculares progresivas y conducentes a grados y títulos. Dentro del mismo informe, se menciona que

La menor cobertura se presenta en la educación media. Aunque entre 2000 y 2005 los matriculados se incrementaron en más 33 mil (sic) y se llegó a cubrir casi un millón de estudiantes, 7 de cada 10 jóvenes en edad de cursar la media no están estudiando. Un poco más de la tercera parte de quienes asisten a $10^{\circ}$ y $11^{\circ}$ grado tienen (sic) por lo menos tres años más de la edad prevista para este nivel. A pesar de que en todos los departamentos la cobertura aumentó, existen brechas entre ellos. En 2004, en Guainía, Guaviare, Vaupés y Vichada sólo 1 de cada 10 jóvenes asistía a este nivel en la edad en que debería hacerlo; en tanto que en Bogotá lo hacían 4 de cada 10.

Es así como para cada nivel de escolaridad y como puede verse en la Figura 3 , el nivel de matriculados muestra una tendencia creciente; sin embargo, en ella persisten algunas inequidades y disparidades regionales. Adicionalmente, y si bien la tendencia de los estudiantes universitarios es creciente, ellos representan una proporción relativamente pequeña de la población total (en promedio $4.8 \%$ del total de matriculados 
para el período 1950-2005). De hecho, su nivel más alto es en 2005, cuando la proporción de estudiantes matriculados en universidad del total de la población es $11.6 \%$.

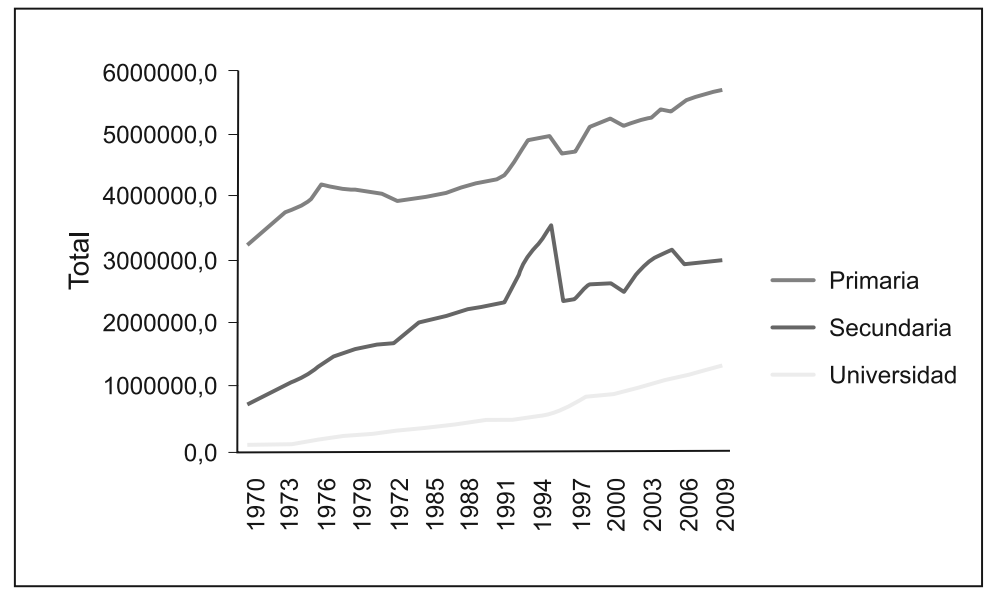

Figura 3. Número de matriculados por nivel de escolaridad.

Fuente: Ministerio de Educación (2009)

\section{La salud en Colombia}

La salud en Colombia ha estado esquematizada de maneras diversas a lo largo de su historia. Según Vivas (2006), a pesar de que la reforma constitucional de 1936 amplió la responsabilidad del Estado en la prestación de servicios de salud, hasta finales de la década del treinta la caridad siguió siendo una forma legítima de distribución de los recursos de salud por fuera del Estado.

De acuerdo con el Departamento Nacional de Planeación (2009), la conducción, la regulación, la modulación de la financiación, la vigilancia de aseguramiento y la armonización de la prestación de los servicios de salud, son responsabilidades del Estado. En el marco del Sistema General de Seguridad Social en Salud, la salud pública tiene entre sus objetivos, fortalecer la capacidad institucional de planificación y gestión, desarrollar las características y condiciones del recurso humano en salud, y elaborar procesos permanentes de investigación dirigida a mejorar las condiciones de salud individuales y colectivas.

De acuerdo con el informe presentado por el Instituto Colombiano de Bienestar Familiar ICBF, la Organización de las Naciones Unidas para la Agricultura FAO y otros (2008), a través de los mapas de la situación nutricional en Colombia, se 
puede decir que "En el análisis de los resultados nutricionales de estos estudios, se identifica un problema de transición nutricional. Se presenta un problema de malnutrición de exceso dentro de los adultos pero de déficit en la población infantil; situación que hace necesario el planteamiento de acciones diferenciales entre grupos poblacionales de acuerdo a la problemática nutricional a abordar".

Otros ámbitos de gran importancia para el desarrollo de las acciones de salud pública, tienen que ver con el seguimiento, evaluación y análisis de la situación de salud (vigilancia epidemiológica), la prevención de la enfermedad y la promo- ción de la salud, la participación de los ciudadanos en los procesos de planeación en salud, el desarrollo de políticas y capacidad institucional de planificación y gestión en materia de salud pública, el desarrollo de recursos humanos y capacitación en salud pública, el saneamiento básico, la investigación, la reducción del impacto de las emergencias y desastres en la salud, entre otras. Las condiciones técnicas y tecnológicas que han mejorado a nivel mundial han llegado a Colombia y las condiciones de regulación muestran sus resultados en variables como la esperanza de vida la cual, como se ve en la Figura 4, muestra un comportamiento creciente.

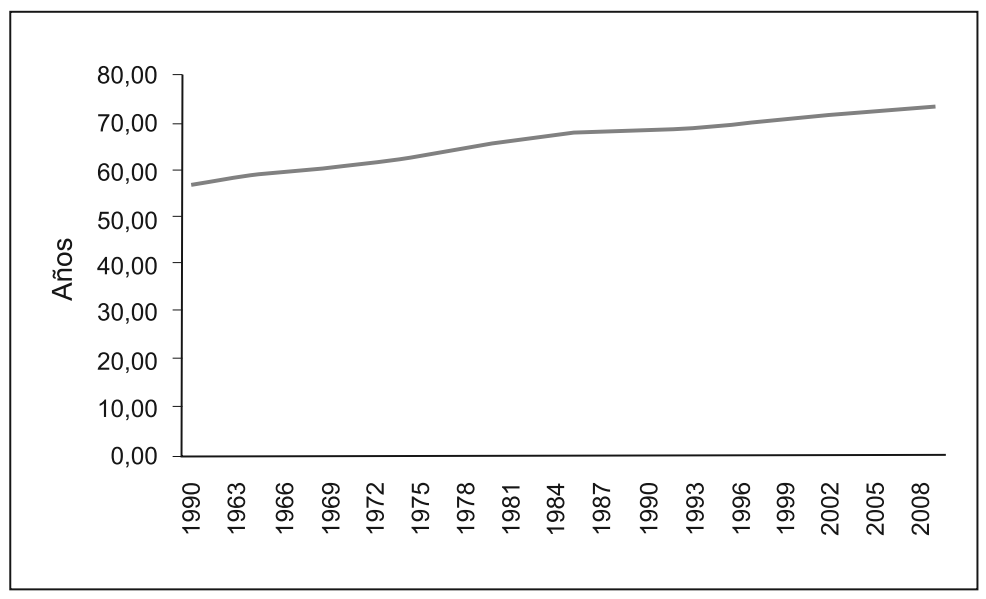

Figura 4. Esperanza de vida.

Fuente: Dane (2009)

\section{Variables por utilizar y primeros resultados}

Al consultar las bases de datos existentes son muy pocas las series anuales de la extensión suficiente para aportar información relevante al modelo empírico, al consultar la base de datos del Banco 
Mundial, anteriormente citada, no existen datos sobre educación de la extensión ni de la temporalidad requerida. En este caso se recurrió a las bases de datos del DANE (2009) de donde se pudo obtener las series de escolaridad y matriculados en primaria secundaria y educación superior.

La escolaridad es el número de alumnos matriculados total, sobre el total de población en edad de estudiar ${ }^{3}$. Esta variable es estacionaria en primeras diferencias de acuerdo con las pruebas aplicadas ${ }^{4}$ y se constituye en un resultado (output) intermedio de educación. La escolaridad está influenciada por las políticas públicas y por las preferencias de la sociedad, pero no necesariamente un aumento en la escolaridad implica un aumento en educación. La razón es que esta variable se mide a partir del número de alumnos matriculados por nivel, luego incluye a aquellos que desertan de las aulas durante el período escolar. Además, una mayor escolaridad no implica que los alumnos realmente aprendan, es decir, la escolaridad no refleja la calidad de la educación que es impartida.

Al no tener otros indicadores, se incluye una nueva variable como alternativa a la escolaridad, que se denomina la pérdida potencial en educación total. Esta serie se obtiene a partir del número de matriculados en primaria, secundaria y educación superior, se restan de los matriculados en secundaria, los de primaria; y, de igual forma, de los matriculados en educación superior, los de secundaria. Luego se promedian ambas diferencias con lo que se obtiene un promedio de alumnos que no accedieron al siguiente nivel de educación. De allí que se denomine pérdida, en la medida en que estas personas no continuarán acumulando capital humano a través de la educación. Esta variable se interpretaría a la inversa de la escolaridad, entre mayor pérdida, menor acumulación de capital humano en términos de educación, luego se esperaría una relación inversa de esta serie frente al PIB per cápita. La palabra potencial se agrega al indicador, pues idealmente se esperaría que los alumnos no abandonen el sistema educativo hasta culminar la educación superior ${ }^{5}$. Para incluir este indicador en términos per cápita, se transforma en cambios y se le resta el cambio porcentual en la población. Esta variable resulta estacionaria en diferencias (I[1]) de acuerdo con las tres pruebas utilizadas.

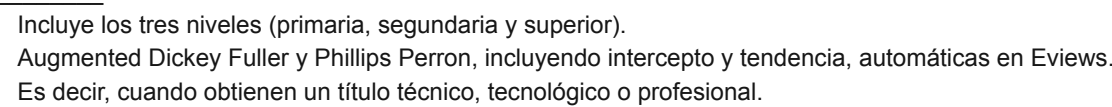




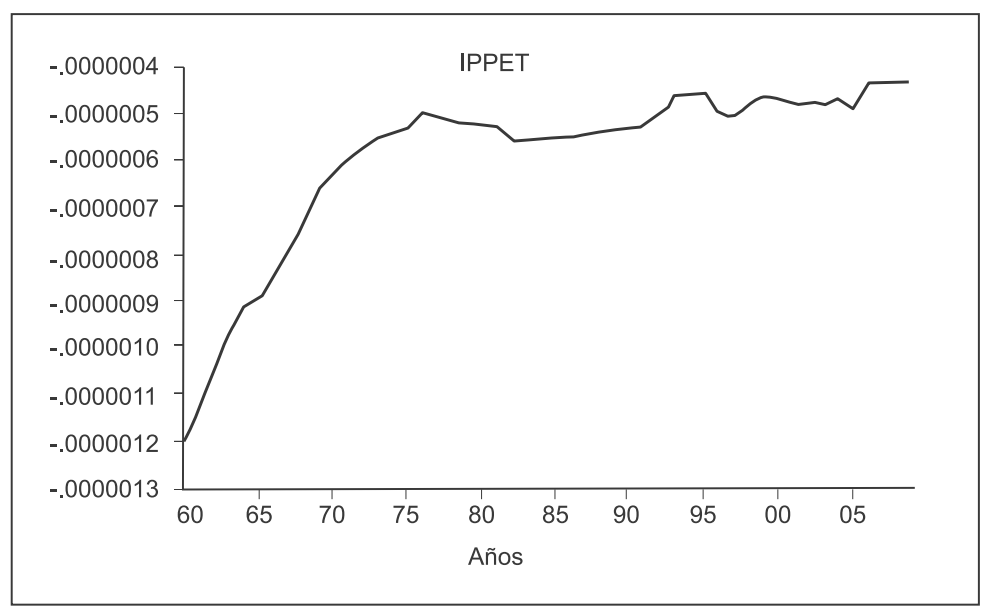

Figura 5. Índice de pérdida potencial en educación.

Fuente: elaboración de los autores

Así como en el caso de la educación, al consultar las bases de datos mencionadas, son muy pocas las series de datos que se encuentran para la extensión requerida. De la base de datos del Banco Mundial se obtuvieron la tasa de nacimientos por cada mil (1000) habitantes $(\mathrm{TN})$, la expectativa de vida al nacer en años (EVN), la tasa de mortalidad por cada mil (1000) habitantes (TM), la tasa de fertilidad total consistente en el número de alumbramientos por mujer (TF).

Al examinar los datos se encontró que la tasa de nacimientos tiene resultados contradictorios en cuanto a la existencia de raíces unitarias. La prueba Augmented Dickey Fuller (ADF) rechaza la hipótesis de raíces unitarias, aun en niveles y también en primeras y segundas diferencias. Por su parte, la prueba Phillips-Perron (PP) no rechaza la hipótesis de raíces unitarias ni en niveles ni en primeras o segundas diferencias. Como tercer criterio se usa la prueba Kwiatkowski-Phillips-Schmidt-Shin $(\mathrm{KPSS})^{6}$, que tiene como hipótesis nula que la serie es estacionaria en tendencia. Cuando se aplica en niveles, el estadístico es mayor que los valores críticos, por lo que se rechaza la hipótesis nula; en diferencias, el estadístico de prueba es pequeño, por lo que se puede aceptar que la serie de la tasa de nacimientos es estacionaria en tendencia en primeras diferencias; por lo tanto, se considera que la serie es estacionaria en primeras diferencias.

$\overline{6}$ Prueba KPSS automática en el paquete eviews. 
La expectativa de vida al nacer también tiene un comportamiento contradictorio en las pruebas. De acuerdo con la ADF, se puede rechazar la hipótesis de raíces unitarias solo en segundas diferencias, la PP en ningún caso rechaza esta misma hipótesis, mientras que la KPSS no rechaza la hipótesis de estacionaridad en primeras diferencias ${ }^{7}$, si se especifican las pruebas de raíz unitaria solo con intercepto, la EVN mantiene resultados contradictorios; pero, en este caso, PP rechaza raíz unitaria en niveles, ADF rechaza en segundas diferencias y KPSS rechaza al $10 \%$ estacionaridad en primeras diferencias y al $1 \%$ en segundas diferencias. La tasa de mortalidad también tiene un comportamiento similar al de la expectativa de vida al nacer. La prueba ADF solo rechaza raíz unitaria en segundas diferencias, la PP, incluyendo intercepto, rechaza en niveles, con intercepto y tendencia no rechaza la hipótesis nula, y la KPSS, incluyendo intercepto, solo acepta estacionaridad en segundas diferencias, cuando se incluye además tendencia no puede rechazar estacionaridad en primeras diferencias.

La tasa de fertilidad muestra resultados variados en las pruebas, la ADF siempre rechaza la hipótesis nula de raíz unitaria, la PP rechaza en primeras diferencias, así como la KPSS que no rechaza la hipótesis de estacionaridad en primeras diferencias. Por lo tanto, se considera que esta serie es estacionaria en primeras diferencias. Las pruebas usadas con las diferentes variables que aproximan el comportamiento de la salud, en general, no concluyen, por lo que se usarán a criterio de los autores teniendo en cuenta que se supondrá la estacionaridad en cierto nivel ${ }^{8}$ y que los resultados que se obtengan usando estas variables podrían ser cuestionables por esta razón; sin embargo, estas series son las únicas existentes, por lo que la investigación se ve obligada a utilizarlas. Además se tendrá en cuenta que la tasa de natalidad es la cantidad de nacimientos con relación a la población total; mientras que la de fertilidad es el número de nacimientos por cada mujer. En este caso, los dos indicadores son bastante cercanos; por ello, se utilizará la tasa de fertilidad, en la que se basan otros estudios como Lucas (1988).

La variable objetivo de este trabajo es el crecimiento económico, que se aproximará con el PIB per cápita (PIBPC), y siguiendo a Lucas (1988), se utilizará en cambios porcentuales. Se incluye también la formación bruta de capital (FBK) como aproximación al factor capital físico $(\mathrm{K})$ también en cambios porcentuales, ambas variables resultaron ser estacionarias en primeras diferencias (I(1)) de acuerdo con las pruebas utilizadas. La frecuencia de todos los datos es anual.

\section{Comprobación empírica: resultados del análisis}

Durante la realización de este estudio, se estimaron varios modelos en los cuales

\footnotetext{
Incluyendo intercepto y tendencia dentro de la prueba.

8 Por lo general, en primeras diferencias.
} 
se conservaron las ideas fundamentales del trabajo. Se busca introducir el capital humano, medido a partir de educación y salud, y se plantea como principio básico un acercamiento a través de la metodología VAR. El análisis comienza con una revisión de las variables en el modelo, evaluando las series que reflejan el capital humano, luego se seleccionan aquellas que teórica y técnicamente tengan un mejor desempeño y se prueba el orden Cholesky, a fin de controlar la estabilidad de los resultados, y, en último término, se explora la posibilidad de cointegración. Siguiendo a Echavarría, López y Misas (2009), en este trabajo se entiende que la metodología VAR es una herramienta idónea en este tipo de trabajos, la cual permite resumir la información contenida en las diferentes series económicas y simular experimentos de política, pero es claro que no está exenta de problemas, algunos de ellos comunes a otras metodologías.

Una vez establecidas las series de datos, y habiendo comprobado que estas series tienen el mismo orden de integración, y realizado varios ensayos de estructura de modelos 9 , para evaluarlas se tienen en cuenta varios criterios:
1. Se deben satisfacer los requerimientos técnicos de especificación del modelo, esto es, que los residuos sean al menos estacionarios, las variables tengan el mismo orden de integración y que el número de rezagos se fije a partir de los criterios AIC y SC, combinados con el propósito del estudio y la teoría.

2. Los resultados arrojados por las descomposiciones de varianza ${ }^{10} \mathrm{y}$ por los impulsos respuesta, deben ser intuitivos, es decir, deben ser coherentes con la teoría en la que se basa el estudio.

3. Si más de una especificación cumple con los criterios 1 y 2 , entre ellas se seleccionará a la mejor de acuerdo con aquellas que reflejen de forma más apropiada la teoría ${ }^{11}$, donde, en su orden, se considerarán más importantes las descomposiciones de varianza, las ecuaciones de cointegración ${ }^{12}$ y los impulsos respuesta ${ }^{13}$.

Ahora bien, aparece una preocupación relacionada con la incidencia que puede tener en el modelo de los choques de corto plazo. Para solucionarlo, se utiliza un concepto sencillo de PIB potencial, aplicando un filtro Hodrick-Prescott sobre la serie del PIB per cápita, con el fin de aislar dichos efectos.

9 Por simplicidad no se incluyen los efectos contemporáneos que, aunque son claros (por ejemplo matricular a un niño en el colegio o pagar un tratamiento médico se constituye en un aporte al PIB), se estaría contabilizando a ambos lados de la ecuación la misma cosa.

10 Se utilizan 10 períodos tanto para las descomposiciones de varianza como para los impulsos respuesta, esta es la configuración que viene automática en e-views, al variar el número de períodos los resultados de las descomposiciones no varían significativamente, por lo que se selecciona esta configuración.

11 Por este criterio se puede entender que será mejor una especificación que arroje la menor cantidad de resultados contraintuitivos.

12 Para los modelos de corrección de error.

13 De acuerdo con Montenegro (2007), se han dejado los gráficos de impulso respuesta como último criterio de selección, pues en caso de que exista correlación entre los errores, no es claro el significado de respuesta Impulso. 
Crecimiento económico en Colombia: una aproximación empírica fundamentada en la perspectiva ...

María Esperanza Cuenca Coral - Ignacio Penagos Montoya

Etapa I: Evaluando las diferentes aproximaciones a salud y educación

Inicialmente se aplica un filtro HodrickPrescott al PIB per cápita y se calcula su cambio porcentual (G1), adicionalmente se le aplican las pruebas de estacionariedad y se concluye que dicha serie no resultó estacionaria, por lo que se descarta su uso; por lo tanto, se debe anotar que los resultados obtenidos contendrán choques de corto plazo no cuantificados, hecho que puede introducir cierto sesgo sobre los mismos.

Entonces, utilizando el cambio porcentual en el PIB per cápita (G2), en esta etapa se configuran seis diferentes modelos donde se utilizan las diferentes combinaciones de las series que han sido obtenidas en la búsqueda de datos. Por salud, se utilizan la expectativa de vida al nacer (SAL1), la tasa de mortalidad (SAL2) y la tasa de fertilidad (SAL3). En teoría, cualquiera de estas tres puede explicar un cambio en la salud, si se incrementa la expectativa de vida, si se reduce la tasa de mortalidad o la tasa de fertilidad ${ }^{14}$. Para reflejar los cambios en educación se usarán dos indicadores, el cambio porcentual en la escolaridad (EDU1) y cambio porcentual de la pérdida potencial en educación total per cápita (EDU2), la acumulación de capital estará representada por el cambio porcentual de la formación bruta de capital (K).

Dado el uso de la metodología VAR, se interpretará el peso de cada factor de producción dentro del PIB per cápita (G2), a través de las descomposiciones de varianza y su concordancia con la teoría a partir de los impulsos respuesta. Además, con el fin de hacer más simple la interpretación en las variables que tienen una relación teóricamente inversa con el PIB, se utilizarán los inversos multiplicativos de las mismas. Los modelos ${ }^{15}$ estimados en general con dos rezagos ${ }^{16}$ muestran a partir de los impulsos respuesta y de las descomposiciones de varianza, que la pérdida potencial en educación total per cápita tiene unos resultados contraintuitivos, por lo que se descarta su uso. Se realizaron varios modelos y se encontró que los más ajustados son los modelos 1,2 y 3 .

Seleccionando entre estos modelos, y de acuerdo con los criterios enunciados, se encuentra que tienen características fortalezas y debilidades; en el modelo 1, los impulsos respuesta (anexo 1) muestran respuestas en su mayoría intuitivas; sin embargo, las descomposiciones de varianza (anexo 2) muestran que la

14 En este caso se supone que entre menos hijos se tienen, es más probable que estos sean más saludables, pues tendrían mayor acceso a servicios de salud.

15 Todos los modelos estimados en el trabajo tienen residuales estacionarios de acuerdo con las pruebas ADF, PP y KPSS, y salvo algunos casos particulares, también son ruido de acuerdo con sus correlogramas y estadísticos $Q$.

16 De acuerdo con las pruebas Akaike Information Criterion y Schwartz Criterion por variable y del modelo, la teoría y maximizando los grados de libertad con el fin de mejorar la verosimilitud de las estimaciones, excepto el modelo 6 que requirió 3 rezagos para obtener residuales estacionarios 
mayor parte del error de pronóstico está explicado por las mismas variables. Lo contrario sucede con el modelo 2 (anexos 3 y 4 respectivamente), se tienen algunos resultados contraintuitivos en los impulsos respuesta, pero la descomposición de varianza le da mayor importancia a otras variables en comparación con los demás modelos estimados. Por estas razones se usarán los modelos 1 y 2 para las siguientes etapas del estudio.

\section{Etapa II: Estimación de las participa- ciones de los factores e interrelaciones}

Utilizando los modelos 1 y 2 se reestiman nuevamente sus descomposiciones de varianza, alterando el orden Cholesky. Estas alteraciones se deben a que no se conoce el verdadero ordenamiento de las variables ${ }^{17}$, sin embargo se considera que el cambio en el PIB per cápita (G2) es la primera de este ordenamiento, al ser la variable objeto de estudio y para evitar imponer un ordenamiento arbitrario. Se vuelven a estimar las descomposiciones de varianza alternando el orden de los tres factores de producción considerados. Los resultados se muestran a continuación en los anexos 5 y 6 , así como en las tablas 1 y 2 , donde se muestran los resultados promedios, mínimos y máximos del aporte de cada una de las variables en la determinación de G2:

La Tabla 1 muestra los resultados del modelo 1, donde, en promedio, la educación (EDU1), medida con el cambio en la escolaridad, es la que más "explica" el crecimiento económico, con cerca del $14 \%$ el capital físico aporta cerca del $1.1 \%$, y la salud, medida a partir de la esperanza de vida al nacer (SAL1), aporta apenas cerca del $0.2 \%$. En la Tabla 2, que muestra los resultados del modelo 2, la educación (EDU1) aporta el mismo $14 \%$ del modelo 1 , pero a diferencia de este último, el capital físico (K) aporta alrededor del $6 \%$ y la salud medida como el inverso de la tasa de mortalidad (SAL2), aporta aproximadamente el $12.3 \%$

Tabla 1

\begin{tabular}{c|c|c|c|c|}
\multicolumn{1}{c}{} & \multicolumn{1}{c}{ G 2 } & \multicolumn{1}{c}{ K } & \multicolumn{1}{c}{ SAL1 } & \multicolumn{1}{c}{ EDU1 } \\
\cline { 2 - 5 } Promedio & 84.79 & 1.09 & 0.20 & 13.93 \\
Mínimo & 83.73 & 0.29 & 0.12 & 11.74 \\
Máximo & 87.84 & 1.30 & 0.38 & 14.61 \\
\cline { 2 - 5 } & & &
\end{tabular}

17 Se fijan dos variables como primera y segunda por una razón práctica más que teórica. El G2 es la variable con que estos modelos permiten medir el crecimiento económico. Y K permite aproximarse a los efectos que tiene la acumulación del capital, variable presente en estos modelos desde Solow y que es sencilla de entender intuitivamente como determinante del crecimiento. 
Crecimiento económico en Colombia: una aproximación empírica fundamentada en la perspectiva ...

María Esperanza Cuenca Coral - Ignacio Penagos Montoya

Tabla 2

\begin{tabular}{c|c|c|c|c|}
\multicolumn{1}{c}{} & \multicolumn{1}{c}{ G 2 } & \multicolumn{1}{c}{ K } & \multicolumn{1}{c}{ SAL2 } & \multicolumn{1}{c}{ EDU1 } \\
\cline { 2 - 5 } Promedio & 67.89 & 5.74 & 12.33 & 14.04 \\
Mínimo & 62.03 & 0.87 & 8.00 & 9.00 \\
Máximo & 81.50 & 9.87 & 14.44 & 17.54 \\
\cline { 2 - 5 } & &
\end{tabular}

Siguiendo a Aghion y Howitt (2009), la parte del crecimiento económico que no es atribuible a los factores de producción se considera explicada por la productividad total de los factores (TFP). En las funciones de producción, la TFP es denotada como el parámetro de estado de la tecnología, $y$, teniendo en cuenta a Whalley y Zhao (2010), en este caso se aproxima la TFP como la proporción de G2 que es explicada por el mismo. Si los factores de producción solo explican entre el $15.21 \%$ y el $32.11 \%$ de acuerdo con los modelos 1 y 2 respectivamente, el restante aporte al crecimiento económico debe estar explicado por la productividad total de los factores, entre el $67.89 \%$ y el $84.79 \%$ del modelo 2 y 1 respectivamente. De acuerdo con los datos y variables obtenidas, el aporte del capital humano medido con salud y educación está entre un $14.13 \%$ y $26.37 \%$.

Se debe tener en cuenta que estos resultados incluyen los sesgos atribuibles a los efectos de choques de corto plazo, a los errores en la medición de las variables, a la aproximación de la salud y la educación por medio de series de datos que no las reflejan plenamente. Llama también la atención la baja participación del capital físico (K) en la determinación del crecimiento económico, en contraste con los aportes de la TFP y del capital humano.

En cuanto a las interrelaciones entre variables, los modelos 1 y 2 muestran que en general esta es baja, de acuerdo con las descomposiciones de varianza de educación y salud, cuyos promedios se muestran en las tablas $3,4,5$ y 6 :

Tabla 3. Salud, modelo 1

\begin{tabular}{c|c|c|c|c|}
\multicolumn{1}{c}{} & \multicolumn{1}{c}{ G 2 } & \multicolumn{1}{c}{ K } & \multicolumn{1}{c}{ SAL1 } & \multicolumn{1}{c}{ EDU1 } \\
\cline { 2 - 5 } Promedio & 22.44 & 4.85 & 69.18 & 3.53 \\
Mínimo & 7.90 & 0.68 & 52.73 & 0.21 \\
Máximo & 31.59 & 6.23 & 91.13 & 10.07 \\
\cline { 2 - 5 } & & &
\end{tabular}

Tabla 4. Educación, modelo 1

\begin{tabular}{c|c|c|c|c|}
\multicolumn{1}{c}{} & \multicolumn{1}{c}{ G 2 } & \multicolumn{1}{c}{ K } & \multicolumn{1}{c}{ SAL1 } & \multicolumn{1}{c}{ EDU1 } \\
\cline { 2 - 5 } Promedio & 2.83 & 1.24 & 0.66 & 95.27 \\
Mínimo & 0.30 & 0.01 & 0.52 & 93.47 \\
Máximo & 4.11 & 1.44 & 0.99 & 99.07 \\
\cline { 2 - 5 } & &
\end{tabular}


Apuntes Cenes Vol. 33, N ${ }^{\circ}$. 58, ISSN 0120-3053

Julio - Diciembre 2014, Págs. 11-44

Tabla 5. Salud, modelo 2

\begin{tabular}{c|c|c|c|c|}
\multicolumn{1}{c}{} & \multicolumn{1}{c}{ G 2 } & \multicolumn{1}{c}{ K } & \multicolumn{1}{c}{ SAL1 } & \multicolumn{1}{c}{ EDU1 } \\
\cline { 2 - 5 } Promedio & 1.22 & 5.57 & 85.48 & 7.73 \\
Mínimo & 0.08 & 1.65 & 58.98 & 0.71 \\
Máximo & 2.30 & 10.57 & 97.54 & 28.34 \\
\cline { 2 - 5 } & &
\end{tabular}

Tabla 6. Educación, modelo 2

\begin{tabular}{c|c|c|c|c|}
\multicolumn{1}{c}{} & \multicolumn{1}{c}{ G 2 } & \multicolumn{1}{c}{ K } & \multicolumn{1}{c}{ SAL1 } & \multicolumn{1}{c}{ EDU1 } \\
\cline { 2 - 5 } Promedio & 2.68 & 2.09 & 6.28 & 88.95 \\
Mínimo & 0.09 & 0.09 & 1.82 & 84.87 \\
Máximo & 3.29 & 3.28 & 8.58 & 98.00 \\
\cline { 2 - 5 } & & &
\end{tabular}

Si bien teóricamente y en otros estudios citados, esta interrelación se ha demostrado, es posible que debido a los posibles sesgos que se han enunciado, los resultados obtenidos no reflejen con claridad la existencia empírica de las mismas.

\section{Etapa III: Cointegración}

Se explora cointegración a partir de los modelos 1 y 2 , se utilizan las variables en niveles y en logaritmos, cambiando la notación por PIB per cápita (LPIBPC), formación bruta de capital (LFBK), esperanza de vida al nacer (LEVN), tasa de mortalidad (LTM) y escolaridad (LESC), al buscar la especificación apropiada en las pruebas de Johannsen se encuentra que al añadir variables se incrementa el número de eigenvalores y al reducirlas se reducen, se eligen dos rezagos para ser consistentes con los modelos estimados anteriormente y no se obtienen resultados significativos.

Si bien hay evidencia de una posible cointegración, las descomposiciones de varianza muestran resultados similares a los promedios hallados en la etapa II, y los impulsos respuesta muestran resultados en general contraintuitivos. Las ecuaciones de cointegración del modelo [1] solo son intuitivas en la escolaridad con coeficiente positivo, y en el modelo dos esta misma variable es la única que tiene un coeficiente negativo que no es acorde con la teoría. La tasa de mortalidad tiene coeficiente negativo y la formación bruta de capital coeficiente positivo, de acuerdo con lo esperado desde la teoría. Los resultados se muestran en el anexo 7.

\section{CONCLUSIONES}

De acuerdo con el objetivo de este trabajo, se ha logrado calcular la proporción del crecimiento económico, medido a partir del PIB per cápita y explicado por la salud y la educación, las estimaciones realizadas reflejan un aporte de entre el $14 \%$ y el $25 \%$ del capital humano y un aporte de la productividad total de los factores de entre el $68 \%$ y el $85 \%$. Por su parte, el capital físico aporta entre un $1 \%$ y $6 \%$ 
aproximadamente. Estos resultados son similares a los obtenidos por otros autores citados anteriormente en términos del aporte del capital humano, pero, por otro lado, difieren en los aportes de la TFP y del capital físico. Existen múltiples sesgos posibles como la forma en que se miden estos dos factores, la carencia de datos o las dificultades técnicas de los mismos, que no permiten afirmar de manera concluyente que los coeficientes estimados reflejen sus verdaderas participaciones. A pesar de ello, coincide, por ejemplo, con los resultados mostrados por Sala-i-Martin en la evidencia empírica de su libro. Allí se muestran las regresiones de Barro (1991), donde al incluir la fracción de la población que asistía a escuelas públicas en primaria y secundaria para el año 1960 en un corte transversal para 114 países, se encuentra un coeficiente positivo y significativo para ambas variables. Es decir que la educación sí tiene una relación positiva con el crecimiento del PIB, lo que nos lleva a pensar que nuestros resultados, a pesar de los sesgos y posibles críticas a la metodología, no son insignificantes.

Las variables usadas en este trabajo son aproximaciones lejanas, en el sentido que existen otros indicadores que desde la teoría podrían aproximar de mejor forma las variaciones en la salud, como indicadores de desnutrición, incidencia de enfermedades, entre otros. De igual manera, es difícil imaginar que la escolaridad, el número de matriculados o indicadores como la pérdida potencial en educación, logren reflejar el verdadero comportamiento de la educación; se debería contar con variables que reflejen la calidad de los graduados, las mejoras en el comportamiento ciudadano, entre otras que en la actualidad no están disponibles. Asimismo, se sugiere la revisión de la metodología con la que se miden las variables utilizadas en este estudio, dado que como resultados de las pruebas practicadas a las mismas y su mera observación parecieran aproximarse a promedios móviles. Es posible que cuando se encuentren disponibles estos indicadores para Colombia o se mejore su medición, se puedan realizar nuevamente las estimaciones y aproximar de forma más apropiada la contabilidad del crecimiento económico. Por otra parte, tampoco es clara la existencia empírica de interrelaciones entre los diferentes factores ni la cointegración entre los factores de producción y el producto interno, hecho que podría ser atribuible a los sesgos ya mencionados.

Sin embargo, el recorrido por los diferentes modelos y estimaciones persiguiendo las conclusiones que se tienen desde la teoría, ha arrojado el resultado satisfactorio de contar con una evidencia que, a pesar de no tener la contundencia necesaria, sí permite presumir que al momento de pensar en crecimiento económico no es prudente enfrentar este problema de forma unívoca, ignorando las interrelaciones existentes entre las variables, sino que es necesario enfrentarlo como un problema multidimensional, que incluya el capital humano. 


\section{REFERENCIAS}

Aghion, P. \& Howitt, P. (2009). The Economics of Growth. Cambridge: MIT Press.

Aghion, P, Howitt P. \& Murtin F. (2010) The Relationship Between Health And Growth: When Lucas meets Nelson-Phelps. NBER Working Papers. Working Paper 15813. Recuperado de http://www.nber.org/papers/w15813

Arrow, (1962). The economic implications of learning by doing. The Review of Economic Studies, 29(3), 155-173.

Barro, R. J. (1991). Economic growth in a cross section of countries (No. w3120). National Bureau of Economic Research.

Becker, G. (1983). El capital humano. Madrid: Alianza.

Bils, M. \& Klenow, P. (2000). Does schooling cause growth? American Economic Review, 90(5), 1160-1183.

Cleuter D. \& Lleras A. (2006). Education and health: evaluating theories and evidence. Nber working paper no. 12352.

Departamento Nacional de Planeación -DNP- (2009). Gran Encuesta Integrada de Hogares. Recuperado de http://www.dnp.gov.co/

Departamento Nacional de Estadística -DANE- (2009). Gran Encuesta Integrada de Hogares. Recuperado de www.dane.gov.co

Echavarría, J. J., López, E., \& Misas, M. (2009). Intervenciones cambiarias y política monetaria en Colombia. Un análisis de VAR estructural. Borradores de Economía, 580.

Gaviria, M. (2007). El crecimiento endógeno a partir de las externalidades del capital humano. Cuadernos de Economía, XXVI (46), 51-73.

Greco (1999). Grupo de estudios del crecimiento económico colombiano. El crecimiento económico colombiano en el siglo xx: aspectos globales. Bogotá: Banco de la República.

Groot, W. \& Maassen, H. (2007, April). The effect of education on health. Economics of Education Review, 26 (2), 186-200.

Jiménez G. \& Simón, B. (2004). Comparación entre indicadores de capital humano en un modelo de crecimiento económico. Ekonomiaz (57).

Kaldor, N. (1955). Alternative theories of distribution. The Review of Economic Studies, 83-100.

Lucas, R. (1988, July). On the mechanics of development planning. Journal of Monetary Economics, 22(1).

Ministerio de Educación Nacional (2001, sep.). Informe nacional sobre la educación en Colombia. En 46 Conferencia Internacional de Educación, Suiza.

Ministerio de Educación (2013). (ver comentario pág. 11). Sistema Educativo Colombiano. Recuperado de http://www.mineducacion.gov.co/1621/w3-article-231235.html. 
Crecimiento económico en Colombia: una aproximación empírica fundamentada en la perspectiva ...

María Esperanza Cuenca Coral - Ignacio Penagos Montoya

Mincer, J. (1993). Human capital and economic growthing: studies in human capital. s.1.: Eduard Elgar Publishing.

Montenegro, A. (2007). Series de tiempo. (5 ed.). Bogotá: Pontificia Universidad Javeriana.

Organización de las Naciones Unidas -ONU-. (2007). World economic situation and prospectives. ONU.

Organización Panamericana de la Salud (2009). Situación de Salud de las Américas. Indicadores Básicos 2009. Recuperado de: http://new.paho.org/hq/dmdocuments/2009/IB_SPA_2009. pdf

Instituto Colombiano de Bienestar Familiar (ICBF), Organización de las Naciones Unidas para la Agricultura y la Alimentación (FAO), Departamento Administrativo Nacional de Estadística (DANE), Universidad de Antioquia (2008). Adaptación y validación interna y externa de la escala Latinoamericana y Caribeña para la medición de seguridad alimentaria en el hogar - ELCSA Colombia. Recuperado de: http://www.fao.org/publications/es/

Posada, C. \& Rubiano, E. (2007). El crecimiento económico internacional en la segunda mitad del siglo xx. ¿Qué factores lo determinaron? Borradores de Economía, Banco de la República, (445).

Romer, P. (1994). The origins of endogenous growth. The Journal of Economic Perspectives, 8 (1).

Sala-I-Martin, X. \& Artadi, V. (1999). Apuntes de crecimiento económico. Barcelona: Antoni Bosch.

Solow, R. (1987). Discurso de aceptación del Premio Nobel. En R. Solow. La teoría del crecimiento. México: Fondo de Cultura Económica.

Schultz, T. (1972). Recursos humanos. En Human capital: cuestiones de política y oportunidades de investigación. s.1.: National Bureau of Economic Research.

Uribe, J. (1994, sep-). Inflación y crecimiento económico en Colombia. Borradores de Economía, Banco de la República (1).

Urrutia, M. \& Posada, C. (2007). Un siglo de crecimiento económico. En: J. Robinson \& M. Urrutia. Economía colombiana del siglo xx, un análisis cuantitativo. Bogotá: Banco de la República.

Vivas, J. (2006). La capacidad hospitalaria en Colombia. Aspectos de su historia en el siglo veinte. En Crecimiento equidad y ciudadanía. Bogotá: CID Universidad Nacional de Colombia.

Whalley, J. \& Zhao, X. (2010). The contribution of human capital to china's economic growth NBER working papers. Working paper 16592. Recuperado de http://www.nber.org/papers/ w16592

Weil, D. (2005). Accounting for the effect to health on economic growth. Rhode Island: Brown University, Department of Economics.

World Bank (2010). World Development Indicators. Recuperado de http://data.worldbank.org/ data-catalog/world-development-indicators 
Apuntes Cenes Vol. 33, N. 58, ISSN 0120-3053

Julio - Diciembre 2014, Págs. 11-44

ANEXO 1

Impulso respuesta del modelo 1

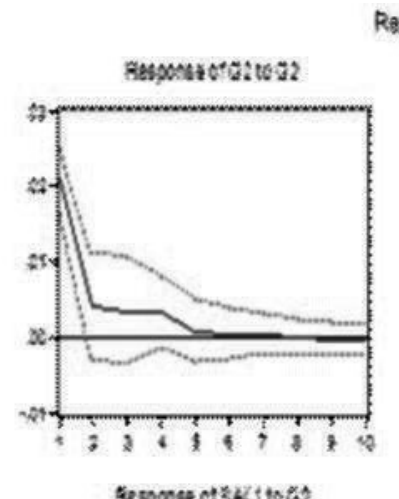

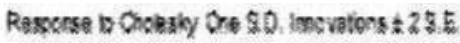
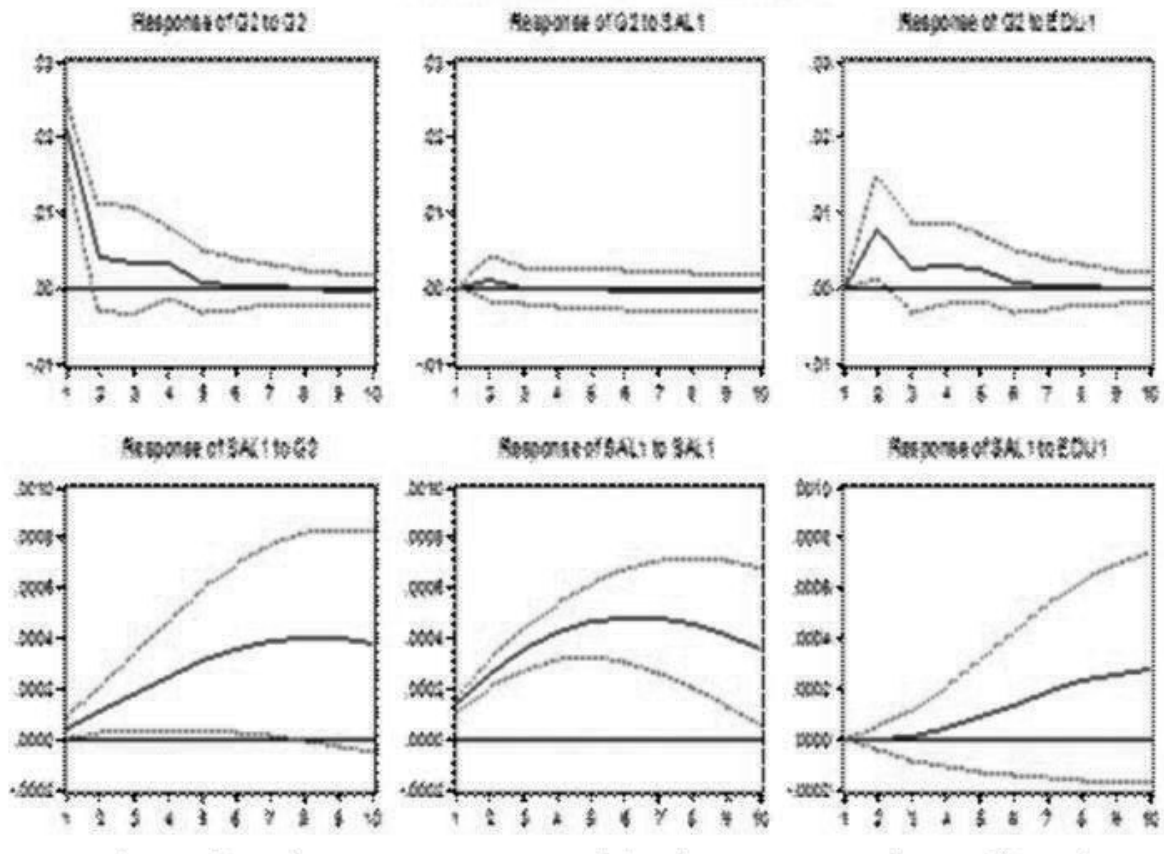

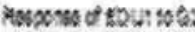
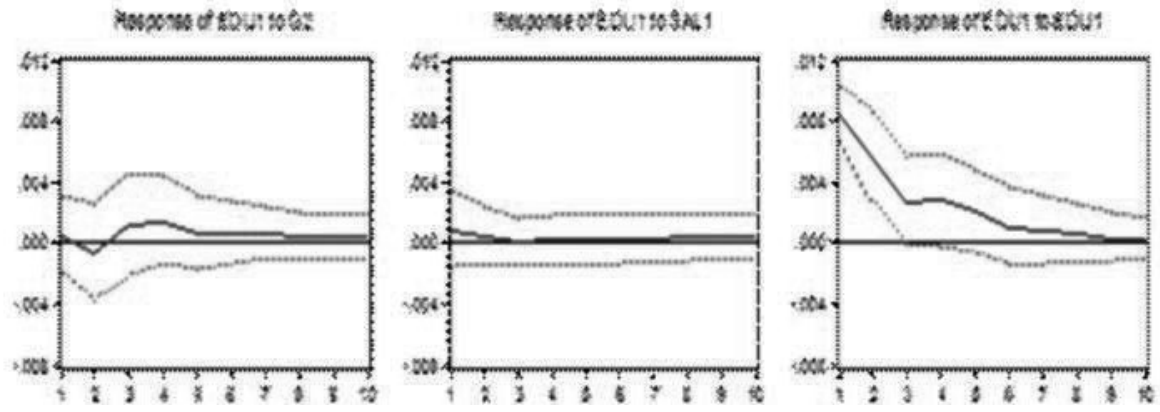
Crecimiento económico en Colombia: una aproximación empírica fundamentada en la perspectiva ... María Esperanza Cuenca Coral - Ignacio Penagos Montoya

ANEXO 2

Descomposición de Varianza del modelo 1

Desc. Var. G

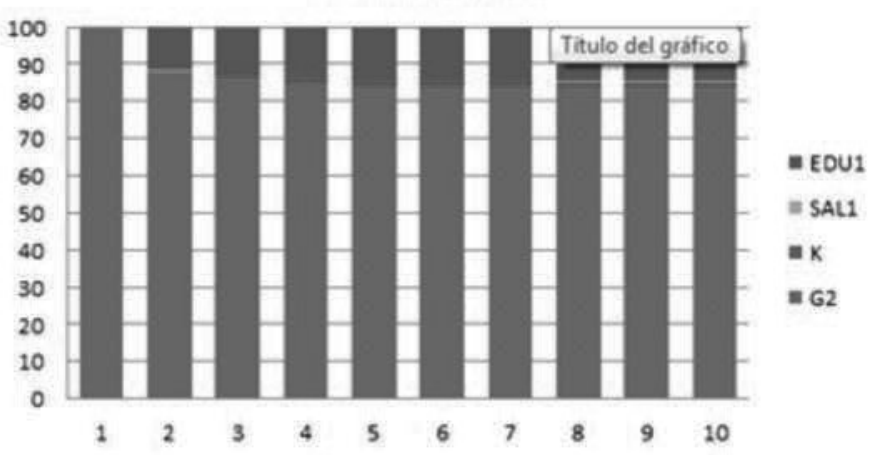

Desc. Var SAL1

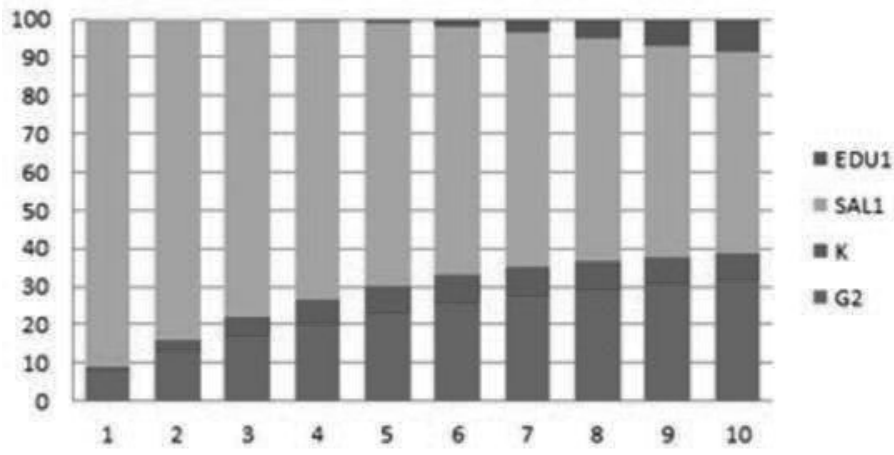

Desc. Var EDU1

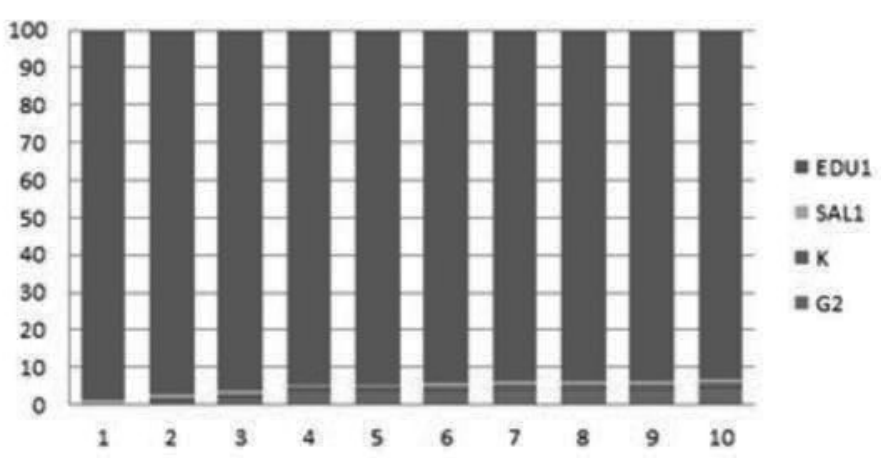


Apuntes Cenes Vol. 33, No. 58, ISSN 0120-3053

Julio - Diciembre 2014, Págs. 11-44

ANEXO 3

Impulsos respuesta del modelo 2
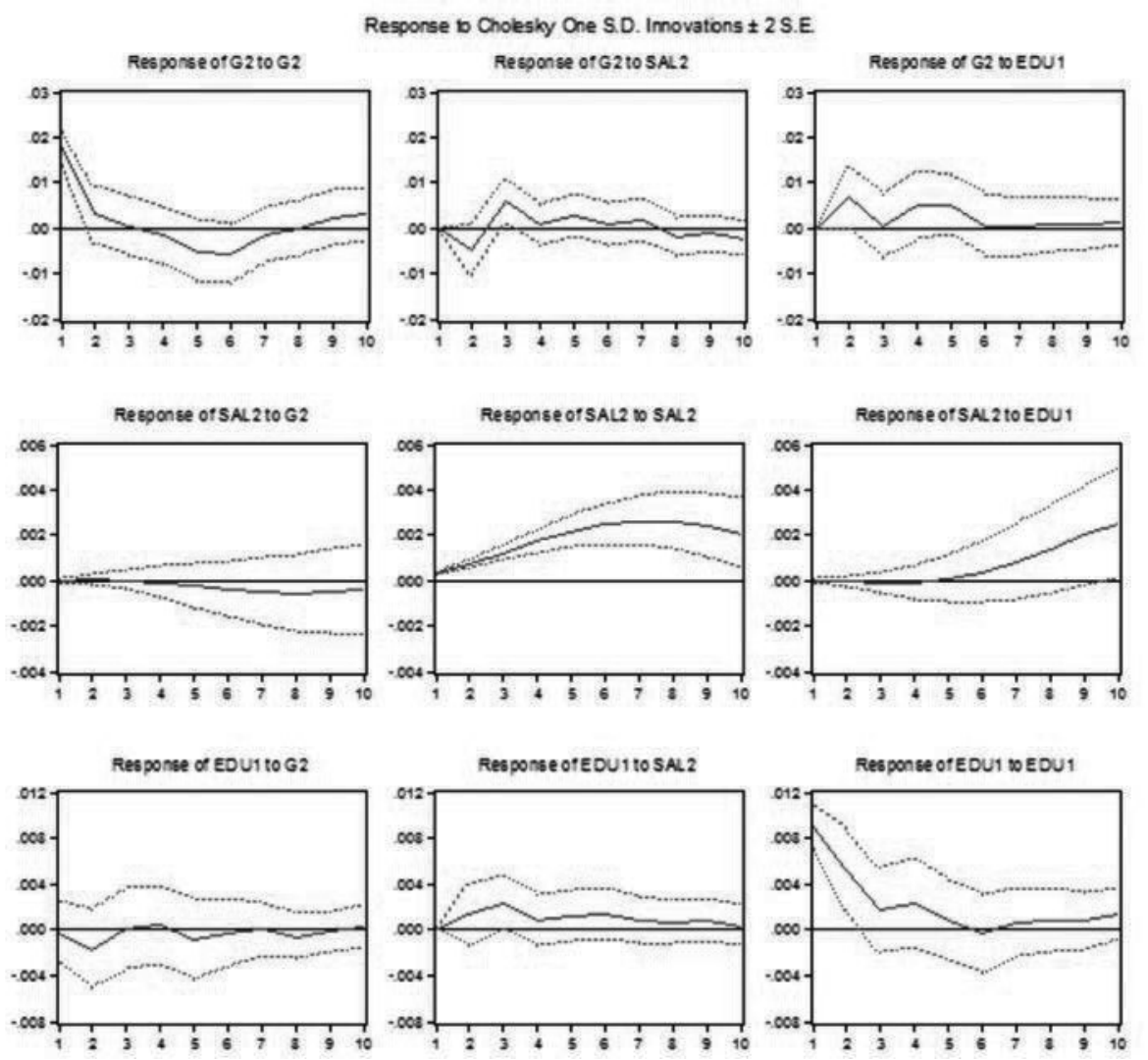
Crecimiento económico en Colombia: una aproximación empírica fundamentada en la perspectiva ... María Esperanza Cuenca Coral - Ignacio Penagos Montoya

ANEXO 4

Descomposición de Varianza del modelo 2

Desc. Var. G

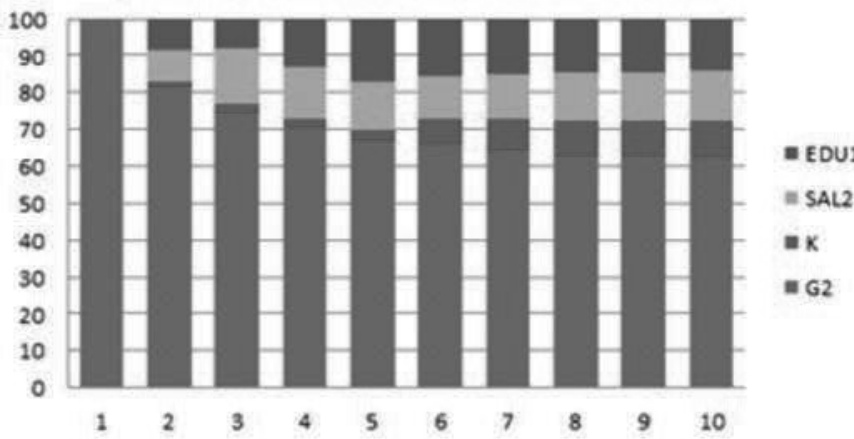

Desc. Var SAL2

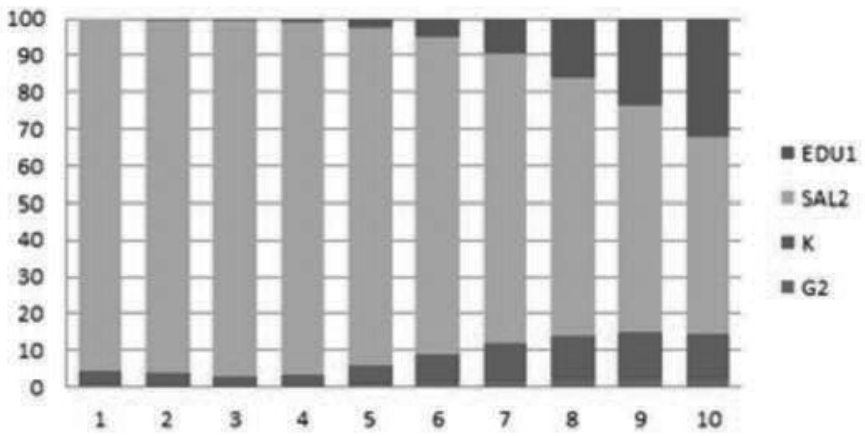

Desc. Var EDU1

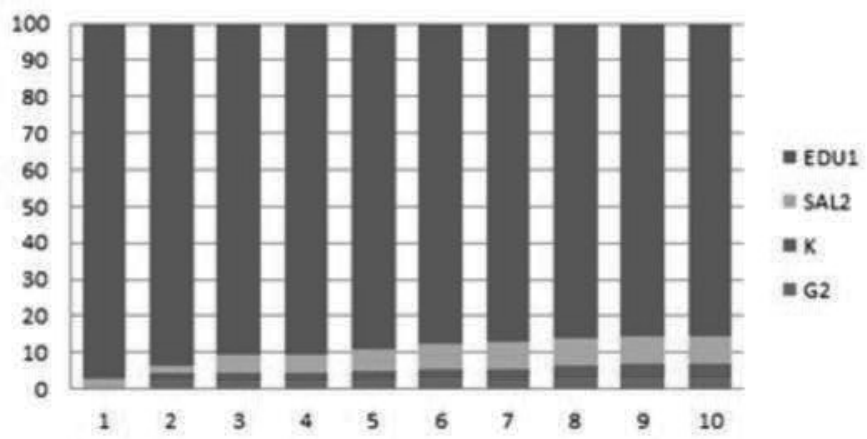


ANEXO 5

Descomposición de varianza del modelo promedio (modelo 1)

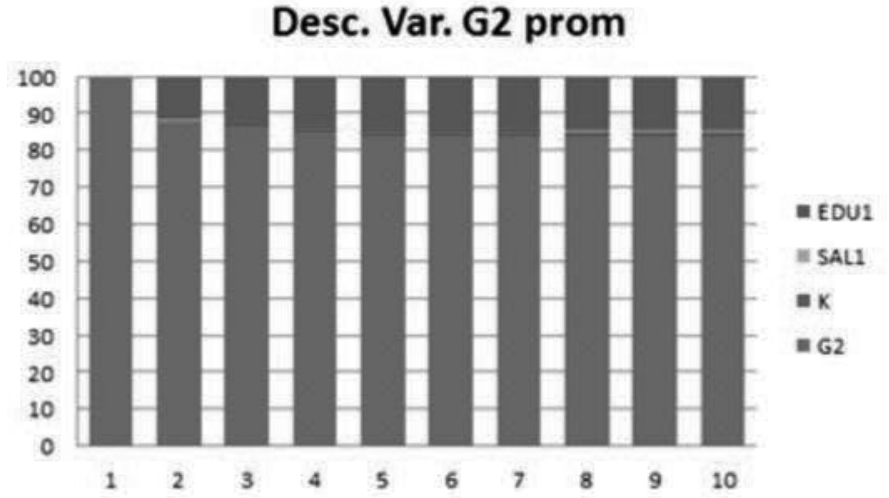

Desc Var. SAL1 prom

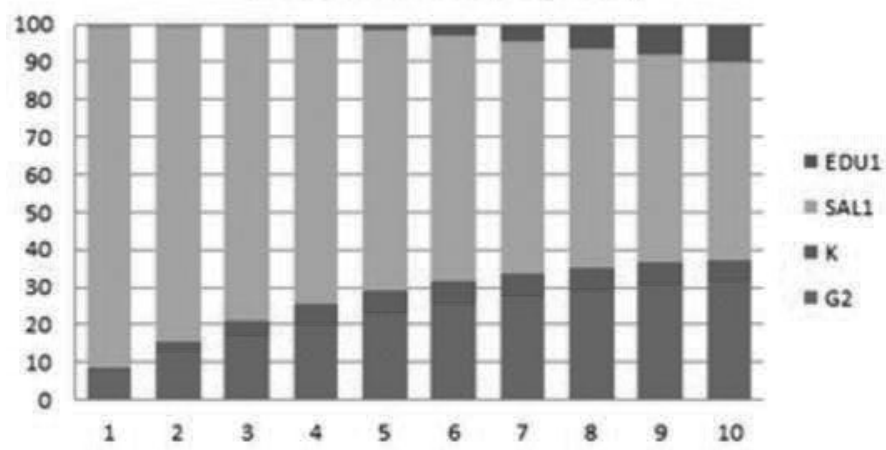

Desc. Var EDU1 prom.

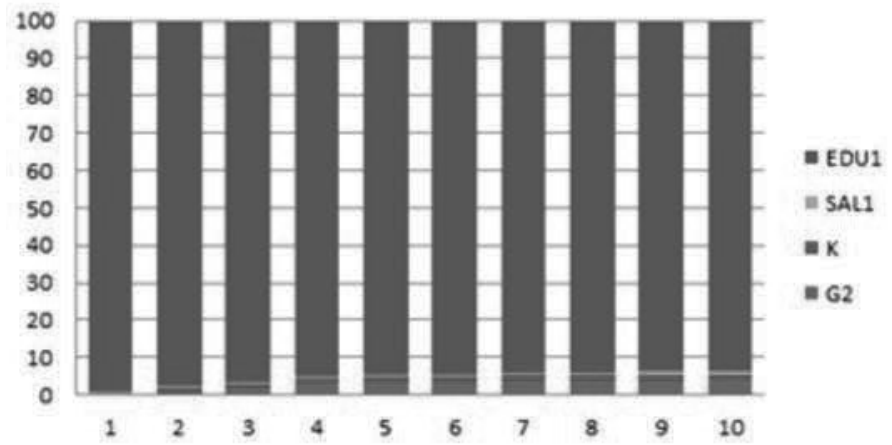


Crecimiento económico en Colombia: una aproximación empírica fundamentada en la perspectiva ... María Esperanza Cuenca Coral - Ignacio Penagos Montoya

ANEXO 6

Descomposición de varianza del modelo promedio (modelo 2)

Desc. Var. G2 prom.

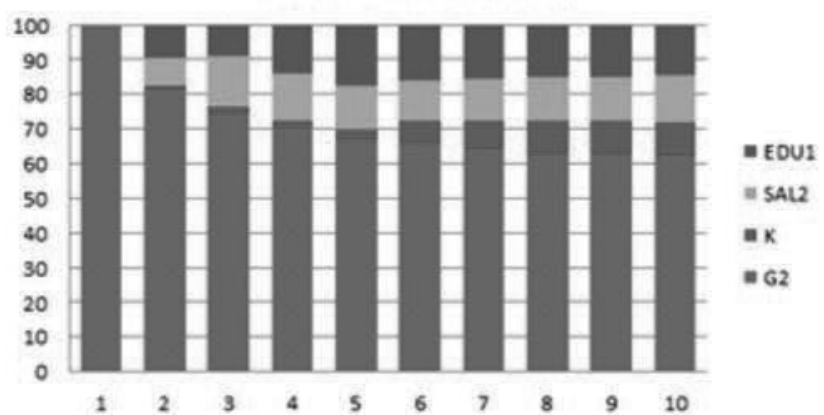

Desc. Var. SAL2 prom.

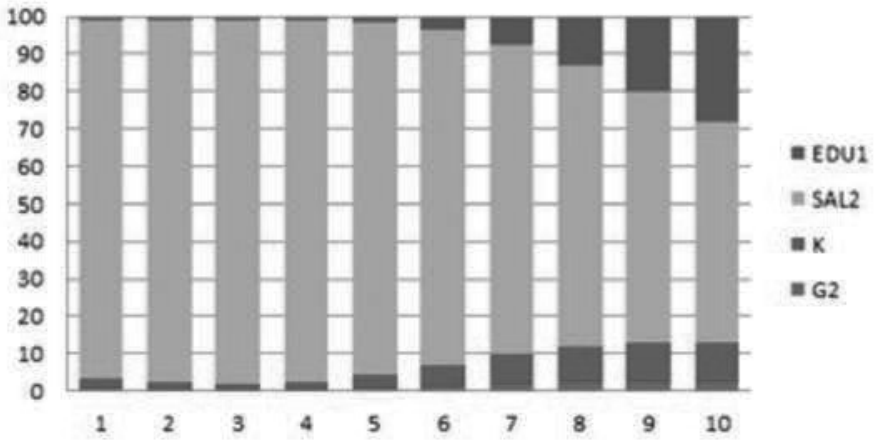

Desc. Var. EDU1 prom.

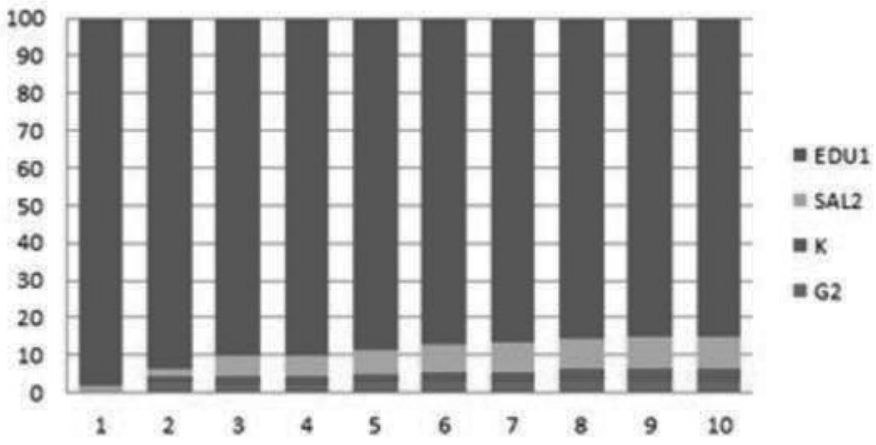


ANEXO 7

Impulso respuesta VEC modelo 1
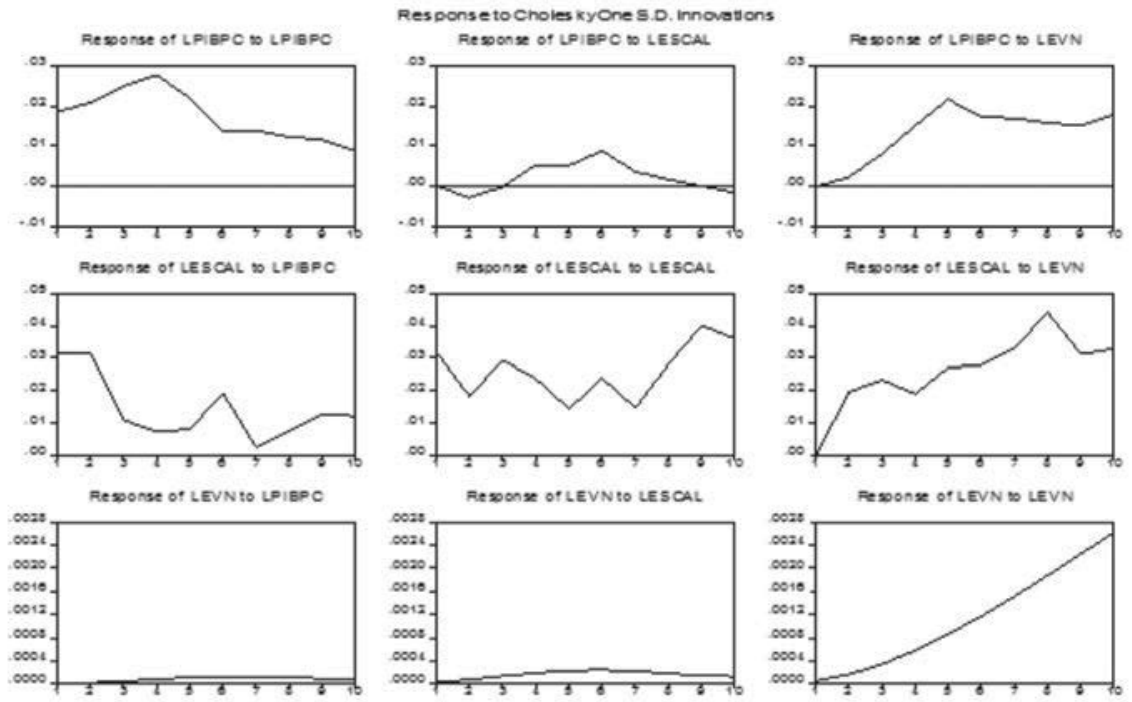

Descomposición de varianza del VEC del modelo 1
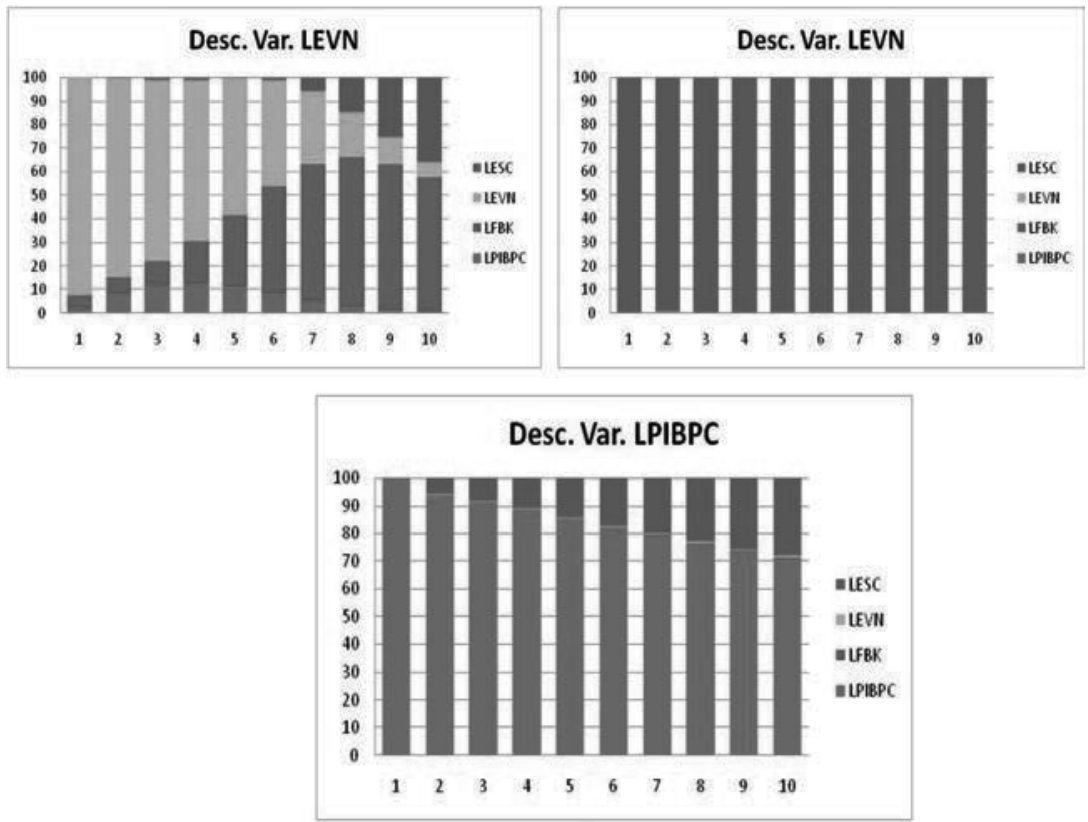
Crecimiento económico en Colombia: una aproximación empírica fundamentada en la perspectiva ...

María Esperanza Cuenca Coral - Ignacio Penagos Montoya

\section{Impulso respuesta VEC modelo 2}
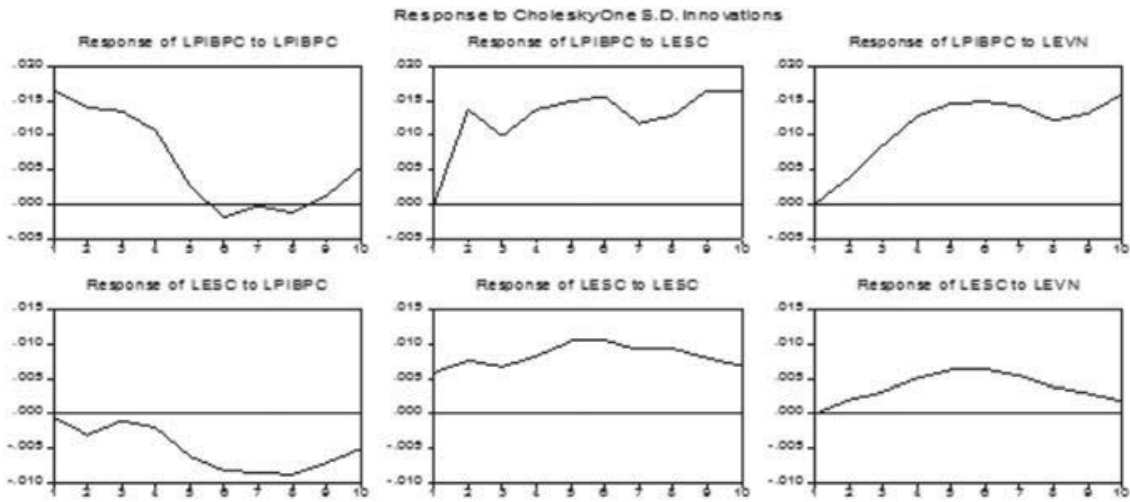

Feson? of LESCTO LEVN
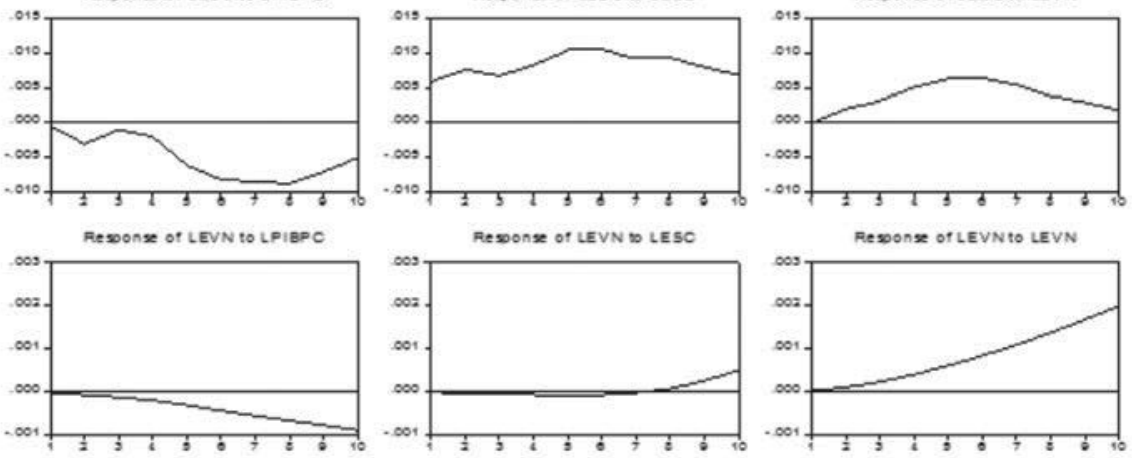

RESONS OF LEVNTO LEVN

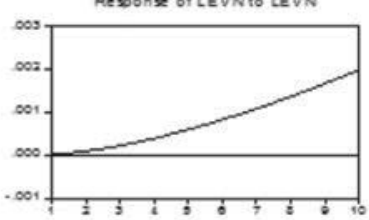

Descomposición de varianza del VEC del modelo 2
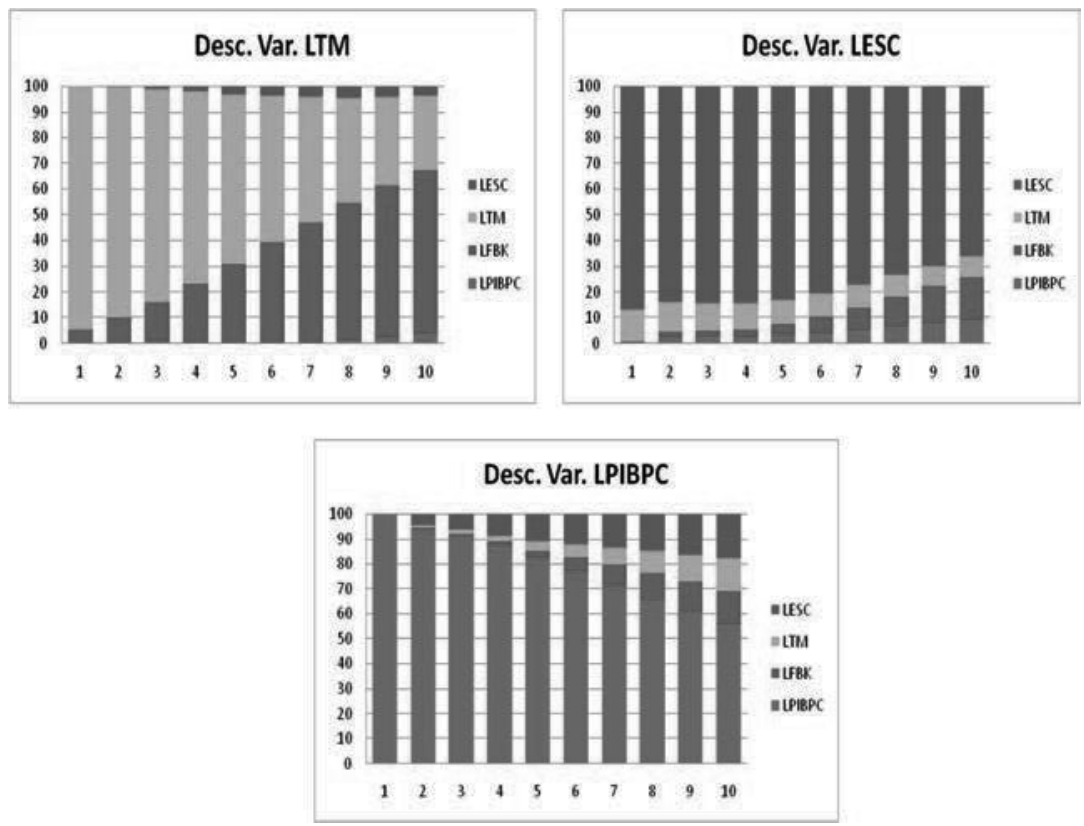